\title{
Mineral Chemistry and Magmatic Differentiation Evidences in the Neshveh Intrusion (NW Saveh, Central Iran)
}

\author{
Reza Keshavarzi', Dariush Esmaili1, Mehdi Rezaei Kahkhaei², Mir Ali Asghar Mokhtari ${ }^{3 *}$, \\ Mehdi Kordlou ${ }^{4}$ \\ ${ }^{1}$ School of Geology, Faculty of Science, University of Tehran, Tehran, Iran \\ ${ }^{2}$ School of Geosciences, University of Shahrood, Shahrood, Iran \\ ${ }^{3}$ Geology Group, Faculty of Science, University of Zanjan, Zanjan, Iran \\ ${ }^{4}$ Geology group, Faculty of Science, Islamic Azad University of Science and Research of Tehran, Tehran, Iran \\ Email: amokhtari@znu.ac.ir
}

Received 19 April 2014; revised 15 May 2014; accepted 23 May 2014

Copyright (C) 2014 by authors and Scientific Research Publishing Inc.

This work is licensed under the Creative Commons Attribution International License (CC BY).

http://creativecommons.org/licenses/by/4.0/

(c) (i) Open Access

\section{Abstract}

Neshveh intrusion which is located in the NW of Saveh City is a part of Sahand-Bazman magmatic arc within the Central Iranian zone. This intrusion consists of quartz-monzogabbro, quartz-monzodiorite, granodiorite and granite that have intruded into the Eocene volcano-sedimentary rocks. This intrusion is medium to high-K calc alkaline, metaluminous, and I-type granitoid. All phases of the Neshveh granitoid are characterized by LREE-rich patterns with high LREE/HREE ratio and negative Eu anomalies. Similarity of patterns suggests a comagmatic source for these rocks and demonstrates the role of magmatic differentiation in their evolution. Clinopyroxene classified as calcic type with varying from clinoenstatite-clinofferosillite to diopside and augite from quartzmonzogabbros to quartz-monzodiorite and granodiorite. Plagioclase composition varies from bytownite and labradorite in quartz-monzogabbros to andesine in quartz-monzodiorites and oligoclase in granodiorites and granites. Core of some plagioclases in granodiorites and granites shows the calcic composition which is labradorite and andesine in granodiorite and andesine in granites. Field investigations along with petrographic and geochemical studies indicate that all phases of the Neshveh intrusion derived from a common magma source as a result of mineral differentiation. Geochemical evidences show smooth differentiation trends in which most of major elements (except $\mathrm{Al}_{2} \mathrm{O}_{3}, \mathrm{~K}_{2} \mathrm{O}$ and $\mathrm{Na}_{2} \mathrm{O}$ ) are negatively correlated with $\mathrm{SiO}_{2}$ and $\mathrm{K}_{2} \mathrm{O}, \mathrm{Ba}, \mathrm{Rb}, \mathrm{Ce}, \mathrm{Nb}$, and $\mathrm{Zr}$ are positively correlated with $\mathrm{SiO}_{2}$. Some elements such as $\mathrm{Na}_{2} \mathrm{O}, \mathrm{Sr}$, Eu and $\mathrm{Y}$ follow curves that reflect crystal fractionation of clinopyroxene, plagioc1ase and hornblende. Furthermore, large volumes of quartz-monzogabbros compared to granites, as well as the lack of mafic enclaves in more evolved rocks, are also indicative of crystal fractionation. Clinopyroxene fractionation was the main control in the evolution of the magmas up to $55 \mathrm{wt} \% \mathrm{SiO}_{2}$. Hornblende took over from $55 \mathrm{wt} \%$

\footnotetext{
"Corresponding author.
}

How to cite this paper: Keshavarzi, R., Esmaili, D., Kahkhaei, M.R., Mokhtari, M.A.A. and Kordlou, M. (2014) Mineral Chemistry and Magmatic Differentiation Evidences in the Neshveh Intrusion (NW Saveh, Central Iran). Open Journal of Geology, 4, 262-288. http://dx.doi.org/10.4236/ojg.2014.46020 
$\mathrm{SiO}_{2}$, resulting in decreasing $\mathrm{Dy} / \mathrm{Yb}$ with increasing silica content in the most siliceous rocks. Fractionation of opaque minerals and apatite throughout the sequence, and the continuous increase in $\mathrm{K}_{2} \mathrm{O}$ and $\mathrm{Ba}$ vs. $\mathrm{SiO}_{2}$ reflect the absence of significant fractionation of biotite and $\mathrm{K}$-feldspar.

\title{
Keywords
}

\author{
Mineral Chemistry, Magmatic Differentiation, Intrusion, Granitoid, Neshveh, Saveh
}

\section{Introduction}

Neshveh intrusion located at about $25 \mathrm{~km}$ in the northwest of Saveh city and is a small part of the Sahand-Bazman magmatic arc in the Alpine-Himalayan orogenic belt. The arc outcrops mainly consist of Eocene-Miocene volcano-sedimentary sequences and associated plutonic rocks typical of calc-alkaline magmatism developed at active continental margins. The arc developed during the closure of the Neotethyan Ocean between Arabia and Eurasia (e.g. [1] [2] [3]), and has been the subject of geophysical, kinematic, and neotectonic studies [4]-[9]. However, little is known about the magmatic activity of the Sahand-Bazman magmatic belt, which was active from Tertiary to Pliocene-Quaternary times (Figure 1) [4] [10] [11].Volcanic rocks are so common in this magmatic belt. These rocks have varieties in composition and tectonic setting which is vary from acidic to basic and con-tinental to shallow marine environments. Acidic-intermediate volcanic and intrusive rocks are widespread in contrast to basic rocks.Intrusive rocks in this magmatic belt show a large range of rock types, dominated by granite and granodiorite but with small amounts of quartz diorite, diorite and gabbro. The Neshveh intrusion was intruded into the volcanic-sedimentary rocks of Eocene (Figure 2) and constitute of quartz monzogabbro, quartz monzodiorite, granodiorite and granite (Figure 3).

Several studies have been carried out in the Saveh region, such as studies of [12] and [13] which were focused on the petrography and Petrochemistry of igneous rocks in the south west of Saveh, volcanic and plutonic rocks in the south of studied area. Previous petrological studies have concentrated mainly on the petrology and tectonic setting of the intermediate-acidic volcanic-plutonic rocks [12]-[15]. In this paper, we combine field and petrography studies with EMPA analysis of the main constitute minerals and whole-rock geochemical data to test whether these various rocks were generated by crystal fractionation or by mixing between basaltic and felsic magmas.

\section{Research Methods}

Our researches in this study consist of two parts: field and laboratory studies. Field studies include identifying the different phases of intrusion, relationship between them and host rock and finally sampling of different phases for laboratory studies. Laboratory investigations include preparing of 90 thin section and petrographic studies. After detailed optical inspection, 8 fresh samples selected for electron microprobe analysis. The major oxide compositions of the minerals (e.g. plagioclase, alkali feldspar, clinopyroxene, amphibole and biotite) in 92 point were determined at mineralogy division of the Iranian Mineral Processing Research Center (IMPRC) using Cameca SX-100 electron microprobe equipped with 5 wavelength-dispersive crystal spectrometers, operating at a $3 \mu \mathrm{m}$ beam diameter, $15 \mathrm{kv}$ accelerating voltage, $15 \mathrm{nA}$ sample current and $60 \mathrm{~s}$ counting time.

Fifteen fresh whole-rock samples representative of the petrological range in the Neshveh intrusion were analyzed for major and trace elements and REEs by ICP and ICP-MS method at ALS Chemex laboratory in Canada. Detection limits range 0.01 - 0.1 wt\% for major oxides, 0.1 - $10 \mathrm{ppm}$ for trace elements, and 0.01 - $0.5 \mathrm{ppm}$ for the rare earth elements.

\section{Regional Geology}

Neshveh region which is located in the northwest of Saveh is a small part of the Sahand-Bazman magmatic belt within the Central Iranian zone. All rocks crops out in this region have Cenozoic age and older rocks don't exposed. Based on Saveh geological map 1:100,000 [17] the main rocks in this area consists of sedimentary, volcanic and intrusive rocks have Paleocene-Eocene age and later (Figure 2). These rocks composed of conglo- 


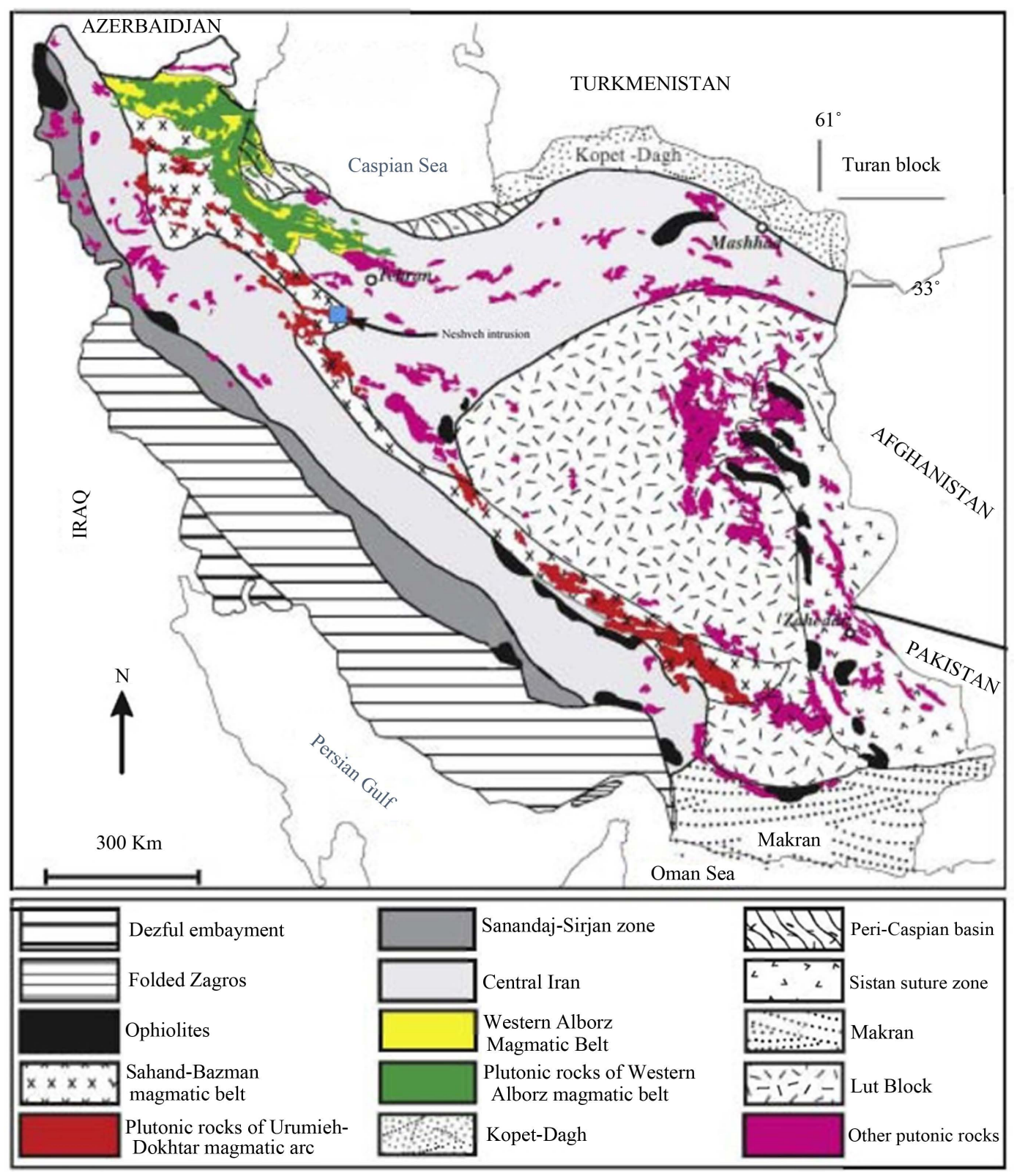

Figure 1. Schematic geological map of Iran, showing the distribution of the sedimentary and structural units and plutonic igneous rocks [after 16]. Sahand- Bazman magmatic arc and studied area is shown on the map.

merate equal to Fajan formation, volcanic-sedimentary rocks of Middle-Upper Eocene consist of intermediateacidic tuffs and lavas, agglomerate and intermediate-basic lavas along with the tuffaceous and sandy limestone and finally Upper Eocene ignimbrite, Lower Red Formation (conglomerate, sandstone, siltstone and red-brown marl), Qom Formation (limestone, marly limestone and marl), Upper Red Formation (conglomerate with red sandstone intercalation), Mio-Pliocene marl, conglomerate equal to Hezar Dareh Formation and quaternary alluvial deposits.

Several granitoid intrusions were intruded into the Eocene volcanic-sedimentary rocks. Dating of some of these intrusions by [12] using K-Ar method on the amphibole, biotite, K-feldspar and whole rock indicate 28 42 Ma (Table 1). The Selijerd granodiorite, Khalkhab tonalite-diorite, Neshveh intrusion (this study) and Neivesht micro-granite are the most important intrusions in the northwest of Saveh. Neshveh intrusion composed of quartz monzogabbro, quartz monzodiorite, granodiorite and granite. In addition to mentioned intrusives, some subvolcanic domes also exist in different parts of the northwest Saveh that have acidic-intermediate composi- 


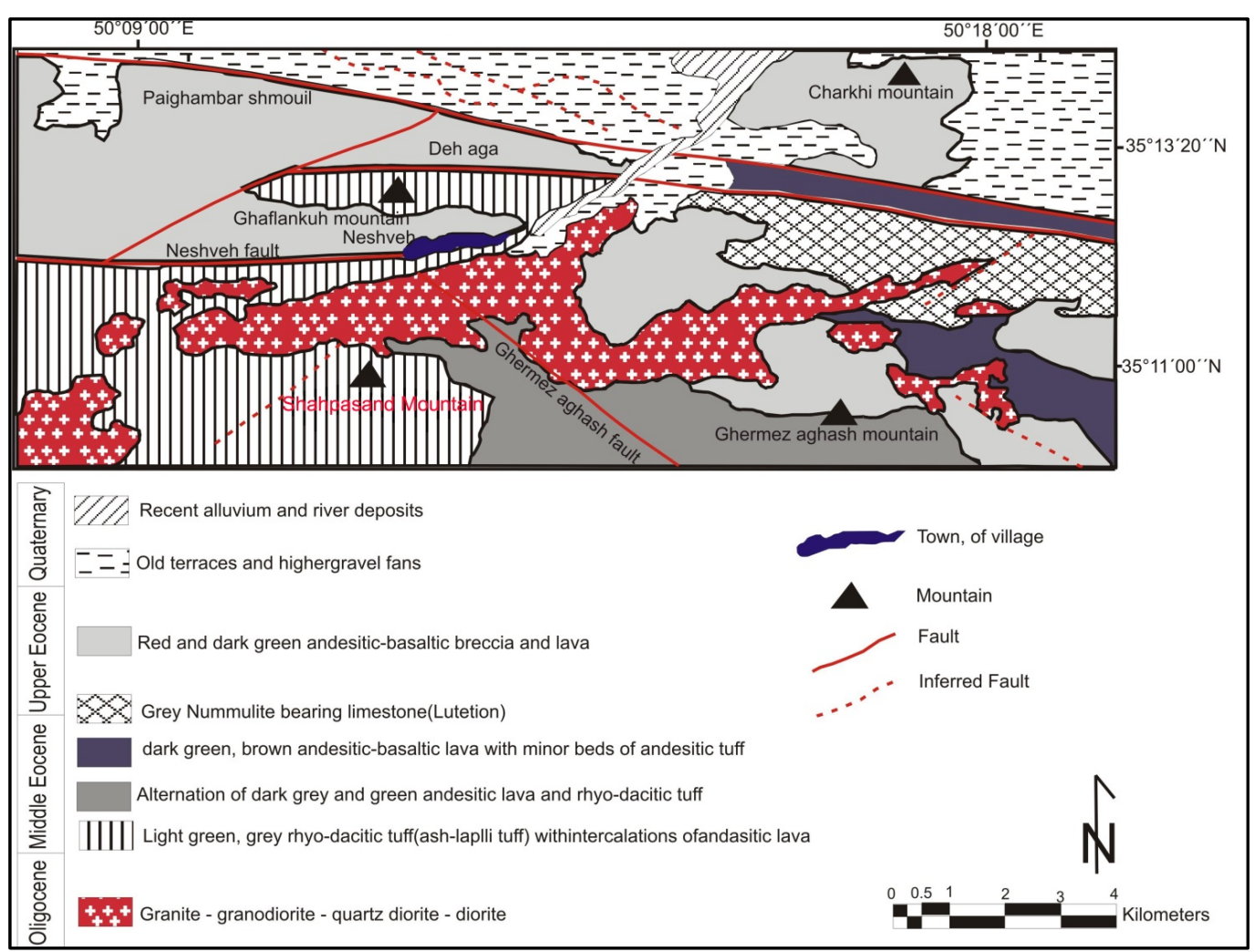

Figure 2. The geological map of studied area based on 1:100,000 geological map of Saveh [17].

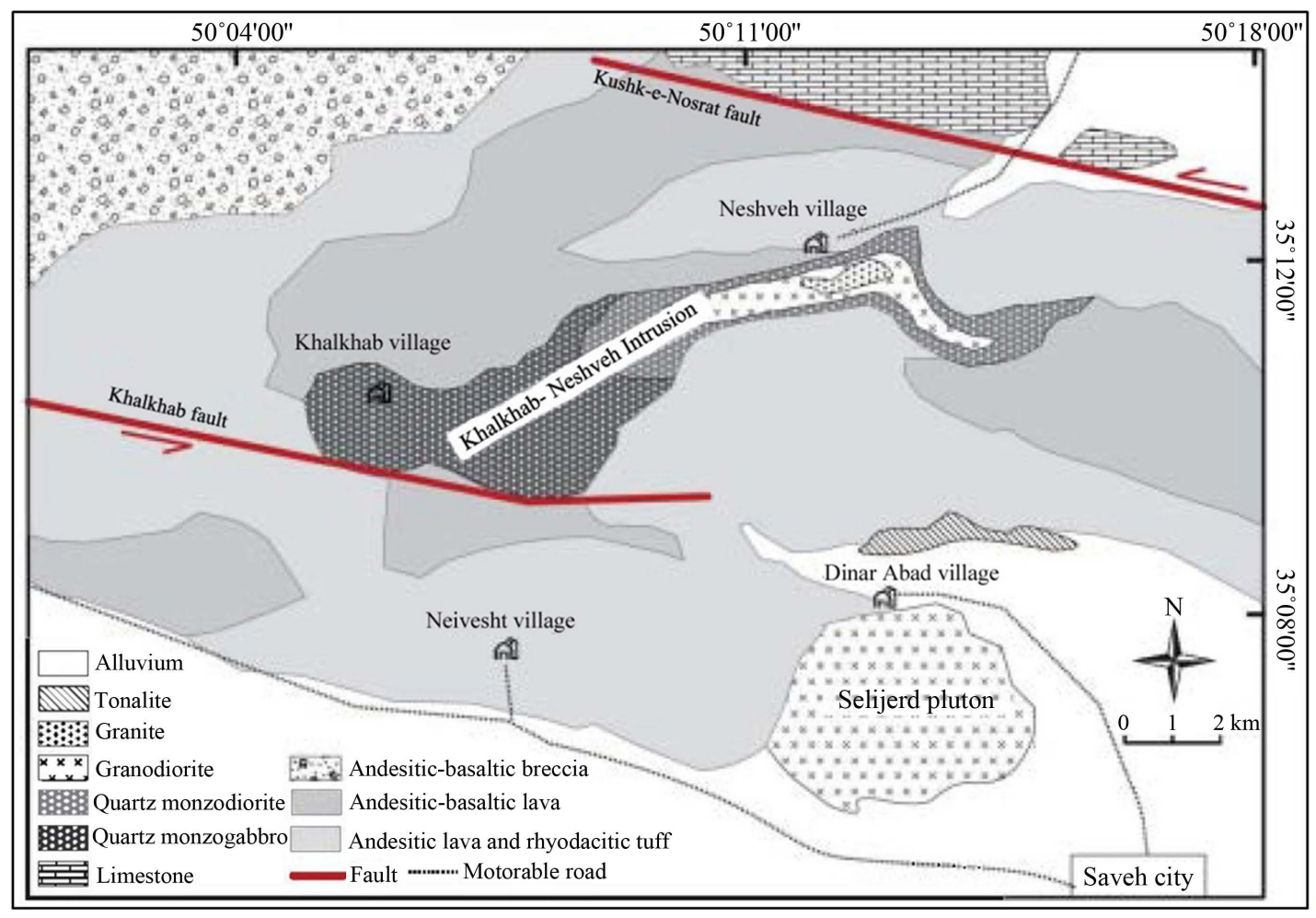

Figure 3. Simplified geological map of NW Saveh intrusions (based on satellite data and the geological map of Saveh in1:100,000 scale [17]). 
Table 1. Modal mineralogical compositions of the Neshveh intrusion rocks.

\begin{tabular}{|c|c|c|c|c|c|c|c|c|c|c|c|c|c|c|c|}
\hline \multirow[b]{2}{*}{ Sample No. } & \multicolumn{5}{|c|}{ Quartz monzogabbro } & \multicolumn{5}{|c|}{ Quartz monzodiorite } & \multicolumn{3}{|c|}{ Granodiorite } & \multicolumn{2}{|c|}{$\underline{\text { Granite }}$} \\
\hline & SK.11 & SK42 & SK18 & SK5 & SK64 & SK58 & SK62 & SN10 & SN11 & SK66 & SN17 & SN15 & SN44 & SN52 & SK56 \\
\hline $\mathrm{SiO}_{2}(\mathrm{wt} \%)$ & 52.1 & 52.2 & 53.2 & 54.7 & 55.5 & 55.1 & 56.7 & 56.5 & 59.6 & 60.8 & 62.5 & 62.7 & 65.1 & 64.9 & 71.2 \\
\hline Quartz & 8.6 & 7.9 & 9.1 & 10.7 & 11.7 & 11.1 & 12.0 & 10.9 & 17.2 & 18.4 & 19.5 & 20.3 & 21.3 & 30.0 & 33.0 \\
\hline K-feldspar & 12.0 & 10.3 & 10.2 & 14.1 & 9.0 & 11.0 & 9.0 & 9.8 & 16.6 & 19.0 & 16.9 & 15.0 & 25.0 & 29.7 & 28.0 \\
\hline Plagioclase & 52.6 & 53.0 & 53.2 & 51.5 & 55.6 & 51.5 & 50.5 & 52.4 & 43.2 & 41.1 & 44.7 & 44.4 & 41.1 & 35.0 & 32.0 \\
\hline Clinopyroxene & 24.6 & 25.7 & 23.9 & 20.8 & 20.2 & 12.1 & 9.5 & 4.5 & 2.7 & 0.0 & 1.2 & 0.4 & 0.0 & 0.0 & 0.0 \\
\hline Hornblende & 0.0 & 0.0 & 0.0 & 0.0 & 0.0 & 6.9 & 13.0 & 14.1 & 13.0 & 16.3 & 11.0 & 12.3 & 11.2 & 2.3 & 4.0 \\
\hline Biotite & 0.0 & 0.0 & 0.0 & 0.0 & 0.0 & 4.1 & 2.5 & 5.0 & 4.5 & 3.0 & 3.6 & 5.4 & 0.0 & 2.2 & 1.2 \\
\hline Apatite & 0.0 & 0.4 & 0.1 & 0.0 & 0.6 & 0.7 & 0.6 & 0.7 & 0.1 & 0.1 & 0.2 & 0.1 & 0.0 & 0.0 & 0.0 \\
\hline Opaque & 2.3 & 2.6 & 3.5 & 2.9 & 2.9 & 2.5 & 3.0 & 2.8 & 2.6 & 2.3 & 2.9 & 2.0 & 1.2 & 0.8 & 0.8 \\
\hline Counted points & 1103 & 1503 & 1938 & 1384 & 1235 & 1698 & 1428 & 1367 & 1251 & 2387 & 1137 & 1600 & 1376 & 1345 & 1212 \\
\hline
\end{tabular}

tion. These domes intruded into the Upper Eocene-Oligocene intrusives. In this base, these sub-volcanic intrusions attributed to the Post Oligocene.

There are some acidic and basic dykes in this area. Basic dykes with a desite-andesitic basalt composition are widespread in comparison to acidic dike with daciteandesite composition. These dikes cut the Eocene volcanic rocks and Oligocene intrusives. In this base, their intrusions have been attributed to the post Oligocene.

The country rocks of the Neshveh intrusion are dominated by andesitic lava and rhyodacitic tuff, andesitic-basaltic lava and andesitic-basaltic breccia associated with limestones [17] (Figure 3). The rhyodacitic tuff is dominantly composed of volcanic grains which, due to explosive acidic eruptions, were ejected as glass shards (now devitrified) and other fragments into a marine environment, forming green minerals such as chlorite and epidote, with various clay minerals [18]. The andesitic-basaltic lavas are dark green and brown in color, with medium-grained phenocrysts, and have minor beds of andesitic tuff. Plagioclase occurs as phenocrysts that range in size from less than $0.2 \mathrm{~mm}$ to several $\mathrm{mm}$. In addition to the ubiquitous laths of plagioclase, the basaltic lavas contain olivine and clinopyroxene, in places altered to iddingsite, and actinolite and chlorite, respectively. The lavas occur as clumps a few $\mathrm{mm}$ to $\mathrm{cm}$ in size within a highly hydrated, oxidized, and altered volcanic matrix, suggesting eruption in a shallow marine environment. In many cases, the exterior margin of these blobs consists of volcanic glass or oxidized material, while the interior parts are more intact, and have preserved an original volcanic texture and structure [18]. The andesitic-basaltic breccia composed of a sequence of hyaloclastic breccia and tuff at the base grade upwards into aphyric lava followed by autoclastic breccia at the top. The tuff grains vary in size from a few $\mathrm{mm}$ to a fewcm, and the smaller grains are replaced by green minerals such as chlorite and epidote, probably because of reaction with water, whereas the larger grains are less altered. Hand specimens show altered phenocrysts of clinopyroxene and plagioclase. Moreover, the olivine basaltic lava at the middle of the sequence and the hyaloclastic breccia at the top might indicate explosive volcanic/volcaniclastic activity under water, probably in a marine basin [18].

The chemical compositions of the volcanic rocks show a calc-alkaline affinity, enrichment in LILEs (Rb, Ba, $\mathrm{Th}, \mathrm{U}$, and $\mathrm{Pb}$ ) and depletion in $\mathrm{Nb}, \mathrm{Ti}$, and $\mathrm{Zr}$ [18]. Significant $\mathrm{U}$ enrichment relative to $\mathrm{Nb}$ and $\mathrm{Th}$ is mainly a result of source enrichment by slab-derived fluids. The results of geochemical modelling suggest a mantle lithosphere source for these volcanic rocks [18].

Two plutonic bodies, Khalkhab-Neshveh and Selijerd intrusions, were intruded into the volcano-sedimentary rocks (Figure 2 and Figure 3). The former comprises quartz monzogabbro, quartz monzodiorite, granodiorite and granite, while the Selijerd pluton in the southern map area consists of tonalite and granodiorite.

\section{Petrography}

The Neshveh intrusion covers an area of about $22 \mathrm{~km}^{2}$ and consists of a wide spectrum of rock types, which 
form high elevation terrains. It was subdivided into two compositional zones: quartz monzogabbro in the West and quartz monzodiorite, granodiorite and granite in the East (Figure 3 and Table 1) based on mineralogy and using the terminology of [19]. The contacts are gradational but the rock types are distinct and easily recognized both in outcrop and in aerial photos and satellite images.

\subsection{Quartz Monzogabbro}

The quartz monzogabbro is poorly exposed in the western part of the Neshveh intrusion and was intruded into the volcanic rocks (Figure 3). It occupies about $50 \%$ of the pluton and is medium to coarse-grained with various textures; some samples show inter-granular and poiklitic textures, while others show hetero-granular texture (Figure 4(A)). The inter-granular texture is constituted by grains of clinopyroxene which occupy the angular interstices between plagioclase crystals. The quartz monzogabbro consists dominantly of plagioclase (51.5 - 55.6 modal \%), clinopyroxene (20.2\% - 25.7\%), K-feldspar (9\% - 14.1\%), quartz (7.9\% - 11.7\%), and subordinate opaque minerals (2.3\% - 3.5\%) and apatite (0.6\%) (Table 1$)$.

Clinopyroxene is a primary mafic phase in the rock and amphibole is absent as a primary phase. In a few samples, especially in the porphyritic quartz monzogabbros, clinopyroxene is replaced completely by actinolite. Plagioclase forms mainly euhedral and lath-shaped crystals. Large plagioclase crystals $(>3 \mathrm{~mm})$ often contain many inclusions of clinopyroxene and opaque minerals (Figure 4(A)). They mainly show zoning, twinning and prismatic-cellular growth. Some large crystals of plagioclase are altered to sericite and clay minerals. Quartz and K-feldspar are anhedral and occupy the interstices between plagioclase tablets, suggesting late crystallization.

\subsection{Quartz Monzodiorite}

The quartz monzodiorite surrounds the granodiorite and granite. To the west, quartz monzodiorite is transitional to more mafic rocks, the quartz monzogabbro while to the east it forms the margin of the pluton (Figure 3). The

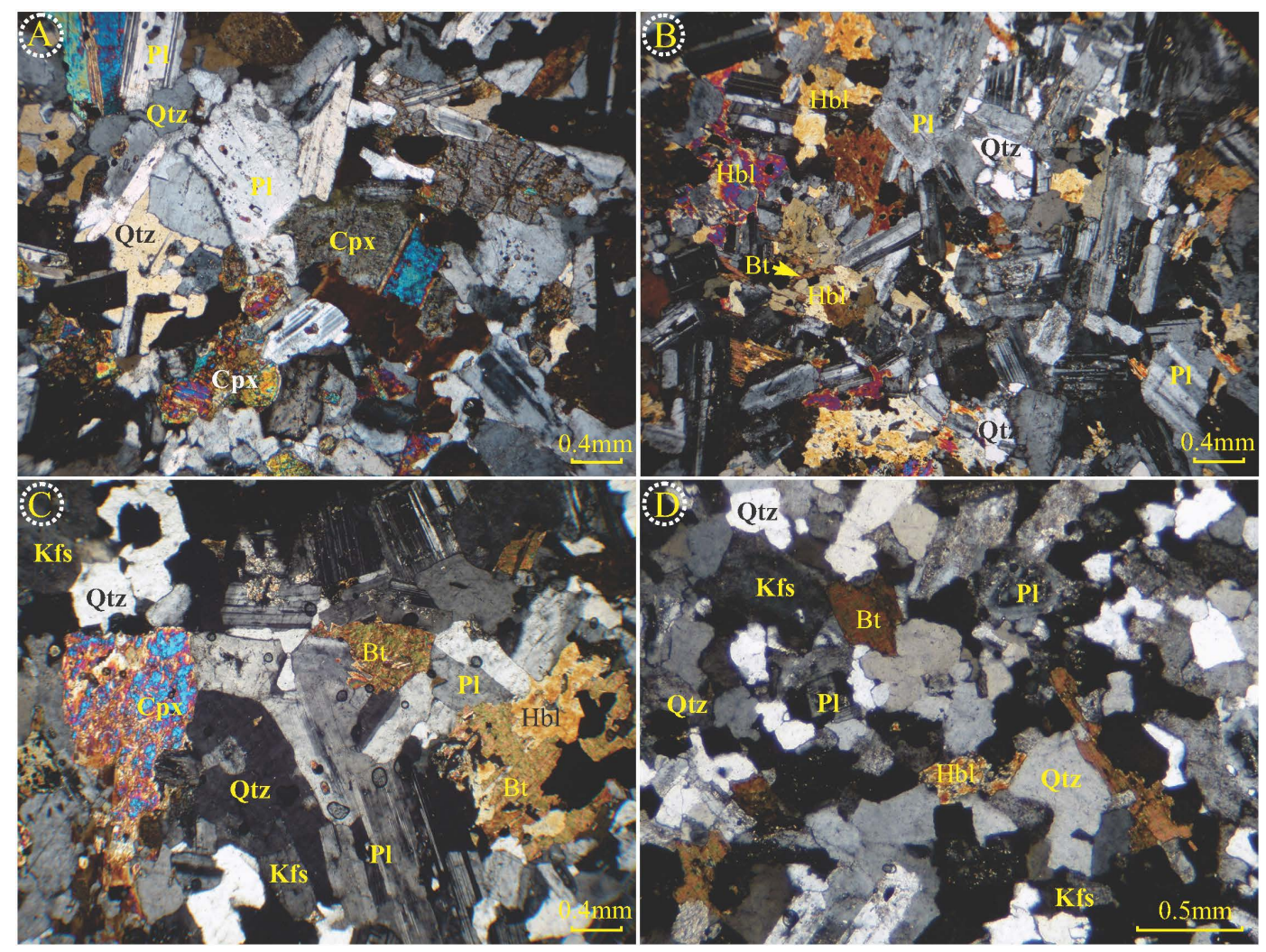

Figure 4. Petrographic features of different rocks from the Neshveh intrusion with granular texture. (A) (B) (C) and (D) are the quartz monzogabbro, quartz monzodiorite, granodiorite and granite, respectively. Abbreviations are after [20]. 
quartz monzodiorite is generally medium-grained and characterized by equigranular texture. It consists dominantly of plagioclase (41.1 - 52.4 modal \%), K-feldspar (9\% - 19\%), quartz (10.9\% - 18.4\%), hornblende (6.9\% - $16.3 \%)$, subordinatesclinopyroxene $(0 \%-12.1 \%)$, biotite $(2.5 \%-5 \%)$ and opaque minerals $(2.3 \%-3 \%)$ (Table 1). Accessory minerals such as titanite and apatite are rare. Clinopyroxene is subhedral and, in some samples, replaced by hornblende, actinolite and biotite aggregates. Hornblende is common in the quartz monzodiorite, where it occurs as euhedral to subhedral and isolated crystals, sometimes accompanied by biotite (Figure 4(B)). Minor interstitial quartz occurs in crude graphic intergrowth with K-feldspar (Figure 5(A)). Opaque minerals are rare but typically form euhedral grains.

\subsection{Granodiorite}

About $20 \%$ of the Neshveh intrusion is constituted by granodiorite, emplaced within the quartz monzodiorite hosted by volcanic rocks. It is relatively homogeneous, white to pale grey massive rocks with a medium-grained texture. The mineral assemblages consist of plagioclase (41.1 - 44.7 modal \%), K-feldspar (15\% - 25\%), quartz (19.5\% - 21.3\%), hornblende (11\% - 12.3\%), biotite ( $0 \%-5.4 \%)$, opaque minerals $(1.2 \%-2.9 \%)$ and clinopyroxene $(<1.2 \%)$, with traces of accessory minerals. Mafic phases are well-formed hornblende and biotite. Biotite forms individual crystals in the granodiorites and is sometimes observed in contact with euhedral to subhedral hornblende crystals (Figure 4(C)). Plagioclase occurs as zoned subhedral crystals, $0.3-1 \mathrm{~mm}$ in diameter and usually twinned. Also, there are some plagioclases that have different composition in the core and rim (Figure 5(B)). Subhedral to anhedral K-feldspar crystals have locally microperthitic texture.

\subsection{Granite}

The white granites are restricted to the centre of intrusion (Figure 3). They are generally medium-grained and have granular to porphyritic textures with megacrysts of plagioclase. They contain plagioclase (32 - 35 al \%), K-feldspar (28\% - 29.7\%) and quartz (30\% - 33\%) with mafic minerals of green hornblende (2.3\% - 4\%) and biotite $(1.2 \%-2.2 \%)$ (Figure 4(D)). There are two kinds of plagioclase in the granitic rocks including medium-grained and large phenocrysts $(\sim 2 \mathrm{~mm})$. Some of K-feldspars are altered, particularly to clay minerals, and occasionally show intergrowth with quartz. Otherwise, quartz is medium-grained and shows undulose extinction. Green hornblende is partially replaced by chlorite and opaque minerals. Magnetite and hematite are the main opaque minerals.

\section{Mineral Chemistry}

As mentioned before, for determining of mineral composition of the Neshveh intrusion, 8 samples from the different parts of this intrusion (quartz monzogabbro, quartz monzodiorite, granodiorite and granite) were selected and 92 points were analyzed by EPMA. Analyzed minerals include plagioclase, alkali feldspar, biotite, amphibole and clinopyroxene. The results of analysis demonstrated in Table 2.
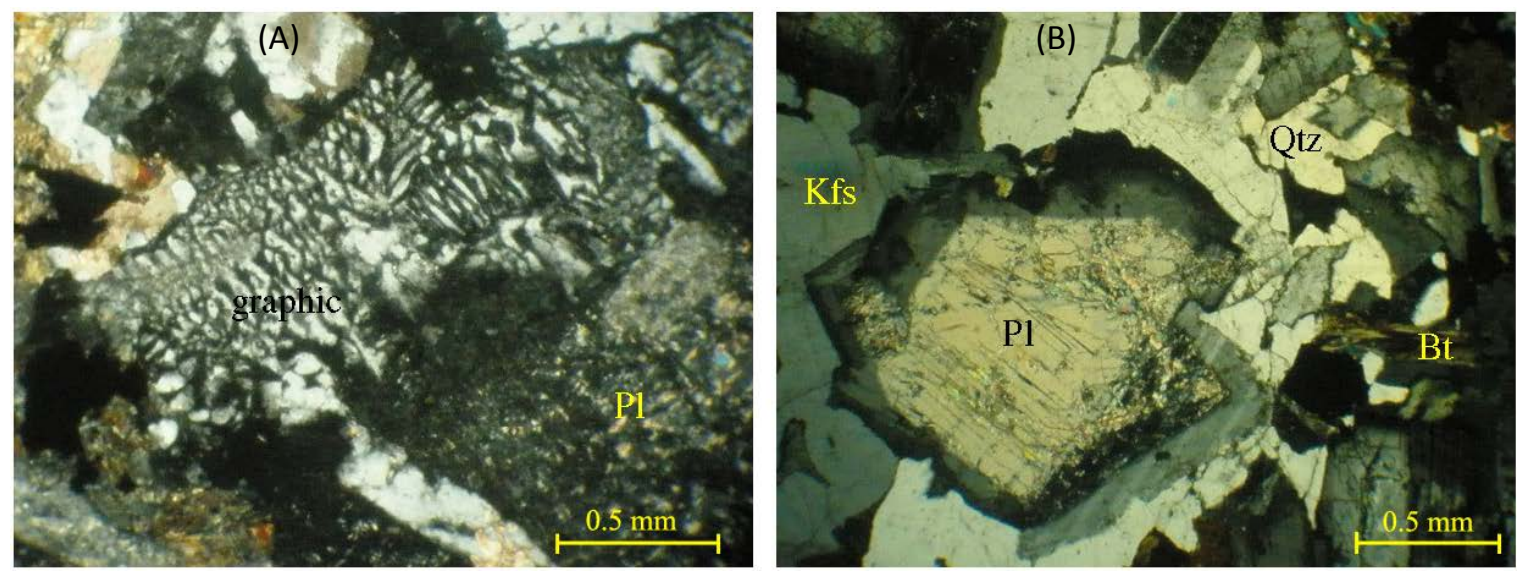

Figure 5. (A) Graphic intergrowth in the quartz monzodiorites; (B) Plagioclase crystal with different composition at the core and rim in the granodiorites. Abbreviations are after [20]. 
Table 2. EPMA analysis of different minerals in the Neshveh intrusion samples.

\begin{tabular}{|c|c|c|c|c|c|c|c|c|c|c|c|c|c|c|c|c|c|c|}
\hline SN 11 & SN 11 & SN 11 & SN 11 & SN 11 & SN 44 & SN 44 & SN 44 & SN 44 & SS63 & SS63 & SK58 & SK58 & SN44 & SN44 & SN44 & SS23 & SS23 & Sample No. \\
\hline Plg & Plg & Plg & Plg & Plg & Plg & Plg & Plg & Plg & Plg & Plg & Plg & Plg & Plg & Plg & Plg & Plg & Plg & Mineral \\
\hline 8 & Core & rim & & Core & rim & & & Core & & & & & rim & core & core & & & Remarks \\
\hline 46.9226 & 50.364 & 58.6802 & 57.0534 & 45.324 & 63.0829 & 56.1899 & 54.6067 & 53.9981 & 62.238 & 59.343 & 56.815 & 56.54 & 65.877 & 55.35 & 53.584 & 60.91 & 63.607 & $\mathrm{SiO}_{2}$ \\
\hline 33.5143 & 30.612 & 25.1047 & 26.4059 & 33.4038 & 22.2742 & 26.7265 & 27.7922 & 28.3969 & 23.118 & 21.402 & 25.153 & 27.176 & 22.486 & 28.222 & 28.004 & 23.789 & 22.839 & $\mathrm{Al}_{2} \mathrm{O}_{3}$ \\
\hline 0.0404 & 0.0374 & 0.035 & 0.0318 & 0.0113 & -0.0012 & 0.0045 & 0.0049 & 0.0081 & 0.028 & 0 & 0.034 & 0.045 & 0 & 0.012 & 0.051 & 0.024 & 0.026 & $\mathrm{TiO}_{2}$ \\
\hline \multirow[t]{2}{*}{0.0307} & 0.04 & 0.0199 & -0.0271 & 0.0361 & 0.0164 & -0.0073 & 0.0036 & 0 & 0 & 0.012 & 0.012 & 0 & 0 & 0.059 & 0.027 & 0.009 & 0 & $\mathrm{Cr}_{2} \mathrm{O}_{3}$ \\
\hline & & & & & & & & & & & & & & & & & & $\mathrm{Fe}_{2} \mathrm{O}_{3}$ \\
\hline 0.2816 & 0.2375 & 0.1681 & 0.3833 & 0.5074 & 0.1668 & 0.3338 & 0.3226 & 0.3309 & 0.141 & 1.038 & 0.235 & 0.288 & 0.178 & 0.286 & 0.368 & 0.123 & 0.079 & $\mathrm{FeO}$ \\
\hline \multirow[t]{2}{*}{0.0059} & 0 & 0.0118 & 0 & 0.0059 & 0 & 0 & 0.0089 & 0 & 0.013 & 0.038 & 0 & 0.006 & 0.003 & 0 & 0 & 0 & 0 & $\mathrm{MnO}$ \\
\hline & & & & & & & & & & & & & & & & & & $\mathrm{NiO}$ \\
\hline 0 & 0 & 0.0086 & 0.005 & 0 & 0 & 0 & 0.0207 & 0.0021 & 0.014 & 0.047 & 0 & 0.015 & 0 & 0.006 & 0.014 & 0 & 0 & $\mathrm{MgO}$ \\
\hline 17.9382 & 14.364 & 7.7469 & 9.3019 & 18.3762 & 4.1344 & 10.0019 & 11.1809 & 11.8173 & 4.719 & 5.447 & 7.525 & 7.024 & 3.017 & 10.663 & 9.906 & 5.175 & 4.832 & $\mathrm{CaO}$ \\
\hline 1.6697 & 3.5645 & 7.0732 & 6.215 & 1.2943 & 9.1062 & 5.9848 & 5.2823 & 4.7885 & 8.389 & 6.295 & 6.272 & 5.573 & 9.702 & 5.801 & 5.767 & 9.017 & 9.56 & $\mathrm{Na}_{2} \mathrm{O}$ \\
\hline 0.0296 & 0.0973 & 0.3518 & 0.3274 & 0.0394 & 0.5253 & 0.2592 & 0.3009 & 0.2372 & 0.665 & 2.256 & 0.456 & 1.204 & 0.652 & 0.209 & 0.22 & 0.312 & 0.348 & $\mathrm{~K}_{2} \mathrm{O}$ \\
\hline 0 & 0 & 0 & 0 & 0 & 0 & 0 & 0 & 0 & 0.012 & 0 & 0 & 0 & 0 & 0 & 0 & 0 & 0.069 & $\mathrm{ZnO}$ \\
\hline 2.154 & 2.315 & 2.647 & 2.574 & 2.119 & 2.815 & 2.546 & 2.483 & 2.455 & 2.779 & 2.778 & 2.631 & 2.583 & 2.853 & 2.486 & 2.472 & 2.730 & 2.790 & $\mathrm{Si}$ \\
\hline 1.813 & 1.658 & 1.334 & 1.404 & 1.840 & 1.171 & 1.427 & 1.489 & 1.522 & 1.216 & 1.181 & 1.372 & 1.463 & 1.148 & 1.494 & 1.522 & 1.256 & 1.180 & $\mathrm{Al}$ \\
\hline 0.001 & 0.001 & 0.001 & 0.001 & 0.000 & 0.000 & 0.000 & 0.000 & 0.000 & 0.001 & 0.000 & 0.001 & 0.002 & 0.000 & 0.000 & 0.002 & 0.001 & 0.001 & $\mathrm{Ti}$ \\
\hline 0.001 & 0.001 & 0.001 & 0.000 & 0.001 & 0.001 & 0.000 & 0.000 & 0.000 & 0.000 & 0.000 & 0.000 & 0.000 & 0.000 & 0.002 & 0.001 & 0.000 & 0.000 & $\mathrm{Cr}$ \\
\hline 0.000 & 0.000 & 0.000 & 0.000 & 0.000 & 0.000 & 0.000 & 0.000 & 0.000 & 0.000 & 0.000 & 0.000 & 0.000 & 0.000 & 0.000 & 0.000 & 0.000 & 0.000 & $\mathrm{Fe} 3+$ \\
\hline 0.011 & 0.009 & 0.006 & 0.014 & 0.020 & 0.006 & 0.013 & 0.012 & 0.013 & 0.005 & 0.041 & 0.009 & 0.011 & 0.006 & 0.011 & 0.014 & 0.005 & 0.003 & $\mathrm{Fe} 2+$ \\
\hline 0.000 & 0.000 & 0.000 & 0.000 & 0.000 & 0.000 & 0.000 & 0.000 & 0.000 & 0.000 & 0.002 & 0.000 & 0.000 & 0.000 & 0.000 & 0.000 & 0.000 & 0.000 & $\mathrm{Mn}$ \\
\hline 0.000 & 0.000 & 0.000 & 0.000 & 0.000 & 0.000 & 0.000 & 0.000 & 0.000 & 0.000 & 0.000 & 0.000 & 0.000 & 0.000 & 0.000 & 0.000 & 0.000 & 0.000 & $\mathrm{Ni}$ \\
\hline 0.000 & 0.000 & 0.001 & 0.000 & 0.000 & 0.000 & 0.000 & 0.001 & 0.000 & 0.001 & 0.003 & 0.000 & 0.001 & 0.000 & 0.000 & 0.001 & 0.000 & 0.000 & $\mathrm{Mg}$ \\
\hline 0.882 & 0.707 & 0.374 & 0.449 & 0.920 & 0.198 & 0.485 & 0.544 & 0.576 & 0.226 & 0.273 & 0.373 & 0.344 & 0.140 & 0.513 & 0.489 & 0.248 & 0.227 & $\mathrm{Ca}$ \\
\hline 0.149 & 0.317 & 0.618 & 0.543 & 0.117 & 0.787 & 0.525 & 0.465 & 0.422 & 0.726 & 0.571 & 0.563 & 0.493 & 0.814 & 0.505 & 0.515 & 0.783 & 0.812 & $\mathrm{Na}$ \\
\hline 0.002 & 0.006 & 0.020 & 0.019 & 0.002 & 0.030 & 0.015 & 0.017 & 0.014 & 0.038 & 0.135 & 0.027 & 0.070 & 0.036 & 0.012 & 0.013 & 0.018 & 0.019 & $\mathrm{~K}$ \\
\hline 0.000 & 0.000 & 0.000 & 0.000 & 0.000 & 0.000 & 0.000 & 0.000 & 0.000 & 0.000 & 0.000 & 0.000 & 0.000 & 0.000 & 0.000 & 0.000 & 0.000 & 0.002 & $\mathrm{Zn}$ \\
\hline 5.013 & 5.015 & 5.003 & 5.005 & 5.020 & 5.008 & 5.011 & 5.014 & 5.001 & 4.993 & 4.984 & 4.977 & 4.966 & 4.998 & 5.024 & 5.029 & 5.041 & 5.035 & Total \\
\hline
\end{tabular}




\section{Continued}

\begin{tabular}{|c|c|c|c|c|c|c|c|c|c|c|c|c|c|c|c|c|c|c|c|}
\hline SN 44 & SN 44 & SS 61 & SS 61 & SS 61 & SS 61 & SN 10 & SN 10 & SN 10 & SN 10 & SN 10 & SN 10 & SN 10 & SK 62 & SK 62 & SK 62 & SK 62 & SK 62 & $\begin{array}{l}\text { SN } \\
11\end{array}$ & $\begin{array}{c}\text { Sample } \\
\text { No. }\end{array}$ \\
\hline $\mathrm{Kf}$ & $\mathrm{Kf}$ & Plg & Plg & Plg & Plg & Plg & Plg & Plg & Plg & Plg & Plg & Plg & Plg & Plg & Plg & Plg & Plg & Plg & Mineral \\
\hline 8 & 8 & 8 & 8 & 8 & 8 & 8 & 8 & 8 & 8 & 8 & 8 & 8 & 8 & 8 & 8 & 8 & 8 & 8 & $\begin{array}{c}\text { No. } \\
\text { oxygen }\end{array}$ \\
\hline & & rim & & Core & & $\begin{array}{l}\text { maybe } \\
\text { rim }\end{array}$ & & $\begin{array}{l}\text { maybe } \\
\text { Core }\end{array}$ & Rim & & & Core & rim & core & rim & core & between & rim & Remarks \\
\hline
\end{tabular}

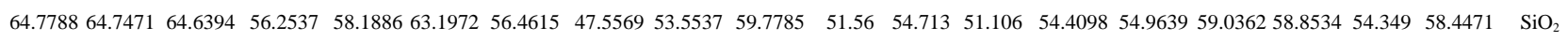

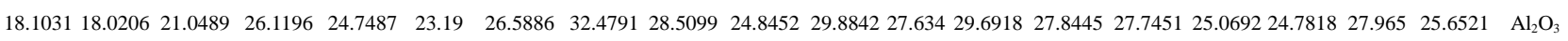

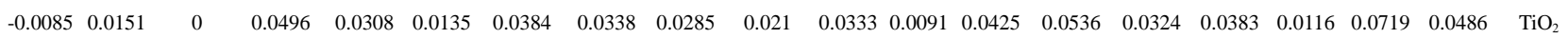
$\begin{array}{lllllllllllllllllllllll}0.0272 & 0 & 0 & 0 & 0 & 0.0397 & 0.0145 & 0 & 0.0323 & 0 & 0 & 0 & 0 & 0 & 0 & 0 & 0 & 0 & 0.04 & \mathrm{Cr}_{2} \mathrm{O}_{3}\end{array}$

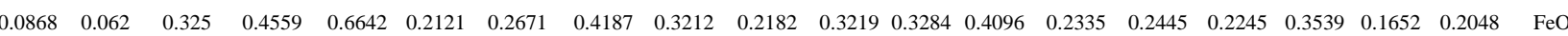

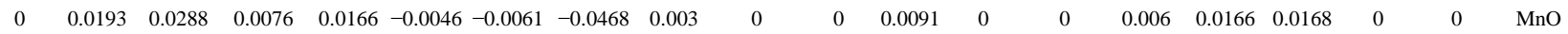

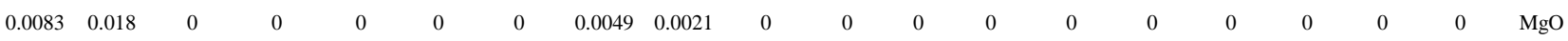

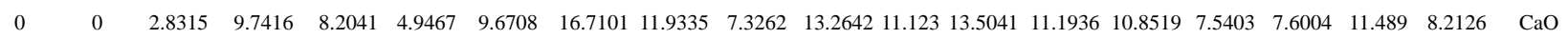

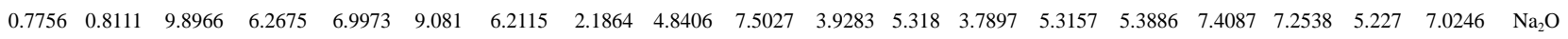

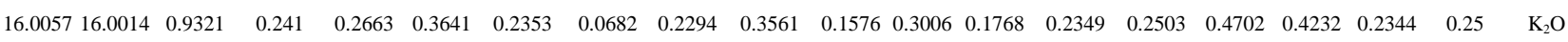

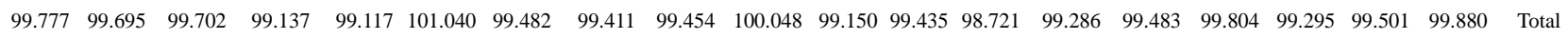

\begin{tabular}{|c|c|c|c|c|c|c|c|c|c|c|c|c|c|c|c|c|c|c|c|}
\hline 3.002 & 3.004 & 2.873 & 2.560 & 2.638 & 2.779 & 2.556 & 2.200 & 2.442 & 2.671 & 2.366 & 2.489 & 2.359 & 2.479 & 2.495 & 2.650 & 2.656 & 2.472 & 2.623 & $\mathrm{Si}$ \\
\hline 0.989 & 0.985 & 1.102 & 1.401 & 1.322 & 1.201 & 1.418 & 1.770 & 1.532 & 1.308 & 1.616 & 1.482 & 1.615 & 1.495 & 1.484 & 1.326 & 1.318 & 1.499 & 1.356 & $\mathrm{Al}$ \\
\hline 0.000 & 0.001 & 0.000 & 0.002 & 0.001 & 0.000 & 0.001 & 0.001 & 0.001 & 0.001 & 0.001 & 0.000 & 0.001 & 0.002 & 0.001 & 0.001 & 0.000 & 0.002 & 0.002 & $\mathrm{Ti}$ \\
\hline 0.001 & 0.000 & 0.000 & 0.000 & 0.000 & 0.001 & 0.001 & 0.000 & 0.001 & 0.000 & 0.000 & 0.000 & 0.000 & 0.000 & 0.000 & 0.000 & 0.000 & 0.000 & 0.001 & $\mathrm{Cr}$ \\
\hline 0.000 & 0.000 & 0.000 & 0.000 & 0.000 & 0.000 & 0.000 & 0.000 & 0.000 & 0.000 & 0.000 & 0.000 & 0.000 & 0.000 & 0.000 & 0.000 & 0.000 & 0.000 & 0.000 & $\mathrm{Fe}^{3+}$ \\
\hline 0.003 & 0.002 & 0.012 & 0.017 & 0.025 & 0.008 & 0.010 & 0.016 & 0.012 & 0.008 & 0.012 & 0.012 & 0.016 & 0.009 & 0.009 & 0.008 & 0.013 & 0.006 & 0.008 & $\mathrm{Fe}^{2+}$ \\
\hline 0.000 & 0.001 & 0.001 & 0.000 & 0.001 & 0.000 & 0.000 & 0.000 & 0.000 & 0.000 & 0.000 & 0.000 & 0.000 & 0.000 & 0.000 & 0.001 & 0.001 & 0.000 & 0.000 & Mn \\
\hline 0.000 & 0.000 & 0.000 & 0.000 & 0.000 & 0.000 & 0.000 & 0.000 & 0.000 & 0.000 & 0.000 & 0.000 & 0.000 & 0.000 & 0.000 & 0.000 & 0.000 & 0.000 & 0.000 & $\mathrm{Ni}$ \\
\hline 0.001 & 0.001 & 0.000 & 0.000 & 0.000 & 0.000 & 0.000 & 0.000 & 0.000 & 0.000 & 0.000 & 0.000 & 0.000 & 0.000 & 0.000 & 0.000 & 0.000 & 0.000 & 0.000 & $\mathrm{Mg}$ \\
\hline 0.000 & 0.000 & 0.135 & 0.475 & 0.398 & 0.233 & 0.469 & 0.828 & 0.583 & 0.351 & 0.652 & 0.542 & 0.667 & 0.546 & 0.528 & 0.363 & 0.367 & 0.560 & 0.395 & Ca \\
\hline 0.070 & 0.073 & 0.852 & 0.553 & 0.615 & 0.774 & 0.545 & 0.196 & 0.428 & 0.650 & 0.349 & 0.469 & 0.339 & 0.469 & 0.474 & 0.645 & 0.634 & 0.461 & 0.611 & $\mathrm{Na}$ \\
\hline 0.946 & 0.947 & 0.053 & 0.014 & 0.015 & 0.020 & 0.014 & 0.004 & 0.013 & 0.020 & 0.009 & 0.017 & 0.010 & 0.014 & 0.014 & 0.027 & 0.024 & 0.014 & 0.014 & $\mathrm{~K}$ \\
\hline 0.000 & 0.000 & 0.000 & 0.000 & 0.000 & 0.000 & 0.000 & 0.000 & 0.000 & 0.000 & 0.000 & 0.000 & 0.000 & 0.000 & 0.000 & 0.000 & 0.000 & 0.000 & 0.000 & $\mathrm{Zn}$ \\
\hline 5.011 & 5.013 & 5.028 & 5.021 & 5.015 & 5.017 & 5.013 & 5.016 & 5.012 & 5.009 & 5.005 & 5.013 & 5.007 & 5.013 & 5.006 & 5.021 & 5.014 & 5.013 & 5.009 & Total \\
\hline
\end{tabular}




\section{Continued}

\begin{tabular}{|c|c|c|c|c|c|c|c|c|c|c|c|c|c|c|c|c|c|c|c|}
\hline SN 11 & SN 11 & SN 44 & SN 44 & SN 44 & SK58 & SK58 & SN44 & SN44 & SS23 & SS23 & SS23 & SS23 & SS 61 & SS 61 & SN 10 & SN 10 & SN 11 & SN 11 & $\begin{array}{c}\text { Sample } \\
\text { No. }\end{array}$ \\
\hline Amph & Amph & Amph & Amph & Amph & Amph & Amph & Amph & Amph & Amph & Amph & Amph & Amph & $\mathrm{Kf}$ & $\mathrm{Kf}$ & $\mathrm{Kf}$ & $\mathrm{Kf}$ & Kf & Kf & Mineral \\
\hline \multirow[t]{2}{*}{23} & 23 & 23 & 23 & 23 & 23 & 23 & 23 & 23 & 23 & 23 & 23 & 23 & 8 & 8 & 8 & 8 & 8 & 8 & $\begin{array}{c}\text { No. } \\
\text { oxygen }\end{array}$ \\
\hline & & & & & & & & & Rim & rim & Core & core & & & & & & & Remarks \\
\hline 50.4978 & 50.6193 & 48.8932 & 47.6056 & 48.693 & 50.839 & 50.603 & 47.326 & 48.156 & 46.643 & 47.212 & 43.986 & 45.497 & 65.6074 & 64.6058 & 64.8122 & 64.3283 & 64.5815 & 64.7526 & $\mathrm{SiO}_{2}$ \\
\hline 3.5934 & 3.6349 & 5.1489 & 6.0613 & 5.0352 & 4.171 & 4.053 & 6.255 & 6.4 & 4.658 & 4.559 & 6.229 & 5.946 & 18.1938 & 18.1698 & 18.2021 & 18.1494 & 17.9769 & 18.192 & $\mathrm{Al}_{2} \mathrm{O}_{3}$ \\
\hline 0.6298 & 0.7658 & 1.2368 & 1.4671 & 1.2606 & 0.771 & 0.782 & 0.88 & 0.917 & 0.555 & 0.554 & 1.003 & 0.932 & 0 & 0 & 0 & 0.0135 & 0.0242 & 0.0549 & $\mathrm{TiO}_{2}$ \\
\hline 0 & 0.0069 & 0.0173 & 0.0398 & 0.0364 & 0 & 0.043 & 0 & 0.013 & 0 & 0 & 0 & 0 & 0 & 0 & 0 & 0 & 0.0343 & 0.0236 & $\mathrm{Cr}_{2} \mathrm{O}_{3}$ \\
\hline 14.9626 & 14.9163 & 14.6506 & 13.4785 & 14.149 & 11.092 & 11.428 & 12.609 & 12.653 & 20.825 & 20.69 & 18.541 & 18.53 & 0.0771 & 0.2353 & 0.0413 & 0.1412 & 0.0327 & 0.2097 & $\mathrm{FeO}$ \\
\hline \multirow[t]{2}{*}{0.6272} & 0.6144 & 0.6036 & 0.4431 & 0.5502 & 0.309 & 0.367 & 0.473 & 0.462 & 0.295 & 0.309 & 0.32 & 0.313 & 0 & 0 & 0.0137 & 0.0227 & -0.0133 & 0.0342 & $\mathrm{MnO}$ \\
\hline & & & & & & & & & & & & & & & & & & & $\mathrm{NiO}$ \\
\hline 14.4393 & 14.2345 & 13.7735 & 14.1976 & 13.967 & 16.684 & 16.472 & 14.916 & 15.376 & 10.38 & 10.257 & 11.496 & 11.343 & 0 & 0.0007 & 0 & 0 & 0.0124 & 0.0335 & $\mathrm{MgO}$ \\
\hline 11.0101 & 11.5278 & 10.9255 & 11.5777 & 11.176 & 9.268 & 9.509 & 11.892 & 11.073 & 12.884 & 11.673 & 12.248 & 12.219 & 0.0112 & 0.008 & 0 & 0.0304 & 0 & 0.186 & $\mathrm{CaO}$ \\
\hline 0.6269 & 0.7486 & 1.2954 & 1.5584 & 1.3221 & 0.672 & 0.827 & 1.613 & 1.604 & 1.206 & 0.999 & 1.645 & 1.625 & 1.7216 & 1.338 & 1.6686 & 1.7059 & 1.1202 & 2.1679 & $\mathrm{Na}_{2} \mathrm{O}$ \\
\hline 0.2815 & 0.2773 & 0.5195 & 0.6801 & 0.4842 & 0.365 & 0.406 & 0.413 & 0.429 & 0.49 & 0.47 & 0.713 & 0.667 & 14.5432 & 14.8771 & 14.6015 & 14.2188 & 15.4851 & 13.6886 & $\mathrm{~K}_{2} \mathrm{O}$ \\
\hline 0 & 0 & 0 & 0 & 0 & 0 & 0 & 0 & 0.066 & 0.034 & 0 & 0 & 0.05 & 0 & 0 & 0 & 0 & 0 & 0 & $\mathrm{ZnO}$ \\
\hline 7.473 & 7.450 & 7.240 & 7.050 & 7.231 & 7.524 & 7.497 & 7.034 & 7.072 & 7.115 & 7.243 & 6.810 & 6.945 & 3.010 & 2.999 & 3.001 & 2.998 & 3.004 & 2.993 & $\mathrm{Si}$ \\
\hline 0.627 & 0.630 & 0.898 & 1.058 & 0.881 & 0.727 & 0.708 & 1.095 & 1.107 & 0.837 & 0.824 & 1.136 & 1.069 & 0.983 & 0.994 & 0.993 & 0.997 & 0.985 & 0.991 & $\mathrm{Al}$ \\
\hline 0.070 & 0.085 & 0.138 & 0.163 & 0.141 & 0.086 & 0.087 & 0.098 & 0.101 & 0.064 & 0.064 & 0.117 & 0.107 & 0.000 & 0.000 & 0.000 & 0.000 & 0.001 & 0.002 & $\mathrm{Ti}$ \\
\hline 0.000 & 0.001 & 0.002 & 0.005 & 0.004 & 0.000 & 0.005 & 0.000 & 0.002 & 0.000 & 0.000 & 0.000 & 0.000 & 0.000 & 0.000 & 0.000 & 0.000 & 0.001 & 0.001 & $\mathrm{Cr}$ \\
\hline 0.000 & 0.000 & 0.000 & 0.000 & 0.000 & 0.000 & 0.000 & 0.000 & 0.000 & 0.000 & 0.000 & 0.000 & 0.000 & 0.000 & 0.000 & 0.000 & 0.000 & 0.000 & 0.000 & $\mathrm{Fe}^{3+}$ \\
\hline 1.851 & 1.835 & 1.814 & 1.669 & 1.757 & 1.372 & 1.415 & 1.567 & 1.553 & 2.656 & 2.654 & 2.400 & 2.365 & 0.003 & 0.009 & 0.002 & 0.006 & 0.001 & 0.008 & $\mathrm{Fe}^{2+}$ \\
\hline 0.079 & 0.077 & 0.076 & 0.056 & 0.069 & 0.039 & 0.046 & 0.060 & 0.057 & 0.038 & 0.040 & 0.042 & 0.040 & 0.000 & 0.000 & 0.001 & 0.001 & 0.000 & 0.001 & Mn \\
\hline 0.000 & 0.000 & 0.000 & 0.000 & 0.000 & 0.000 & 0.000 & 0.000 & 0.000 & 0.000 & 0.000 & 0.000 & 0.000 & 0.000 & 0.000 & 0.000 & 0.000 & 0.000 & 0.000 & $\mathrm{Ni}$ \\
\hline 3.183 & 3.121 & 3.038 & 3.132 & 3.089 & 3.678 & 3.635 & 3.302 & 3.363 & 2.359 & 2.344 & 2.651 & 2.579 & 0.000 & 0.000 & 0.000 & 0.000 & 0.001 & 0.002 & $\mathrm{Mg}$ \\
\hline 1.745 & 1.817 & 1.733 & 1.836 & 1.777 & 1.469 & 1.509 & 1.893 & 1.741 & 2.105 & 1.918 & 2.031 & 1.997 & 0.001 & 0.000 & 0.000 & 0.002 & 0.000 & 0.009 & $\mathrm{Ca}$ \\
\hline 0.180 & 0.214 & 0.372 & 0.447 & 0.380 & 0.193 & 0.237 & 0.465 & 0.456 & 0.356 & 0.297 & 0.494 & 0.481 & 0.153 & 0.120 & 0.150 & 0.154 & 0.101 & 0.194 & $\mathrm{Na}$ \\
\hline 0.053 & 0.052 & 0.098 & 0.128 & 0.092 & 0.069 & 0.077 & 0.078 & 0.080 & 0.095 & 0.092 & 0.141 & 0.130 & 0.851 & 0.881 & 0.862 & 0.845 & 0.918 & 0.807 & $\mathrm{~K}$ \\
\hline 0.000 & 0.000 & 0.000 & 0.000 & 0.000 & 0.000 & 0.000 & 0.000 & 0.007 & 0.004 & 0.000 & 0.000 & 0.006 & 0.000 & 0.000 & 0.000 & 0.000 & 0.000 & 0.000 & $\mathrm{Zn}$ \\
\hline 15.260 & 15.282 & 15.407 & 15.543 & 15.422 & 15.157 & 15.217 & 15.592 & 15.541 & 15.629 & 15.476 & 15.822 & 15.719 & 5.000 & 5.004 & 5.008 & 5.002 & 5.012 & 5.009 & Total \\
\hline
\end{tabular}




\section{Continued}

\begin{tabular}{|c|c|c|c|c|c|c|c|c|c|c|c|c|c|c|c|c|c|c|}
\hline SK 62 & SK 62 & SN 10 & SN 10 & SN 10 & SN 10 & SK 62 & SK 62 & SN 11 & SK58 & SK58 & SS 61 & SS 61 & SS 61 & SS 61 & SN 10 & SN 10 & SN 10 & $\begin{array}{c}\text { Sample } \\
\text { No. }\end{array}$ \\
\hline Bt & $\mathrm{Bt}$ & $\mathrm{Bt}$ & $\mathrm{Bt}$ & $\mathrm{Bt}$ & $\mathrm{Bt}$ & $\mathrm{Bt}$ & $\mathrm{Bt}$ & $\mathrm{Bt}$ & $\mathrm{Bt}$ & $\mathrm{Bt}$ & Amph & Amph & Amph & Amph & Amph & Amph & Amph & Mineral \\
\hline 22 & 22 & 22 & 22 & 22 & 22 & 22 & 22 & 22 & 22 & 22 & 23 & 23 & 23 & 23 & 23 & 23 & 23 & $\begin{array}{c}\text { No. } \\
\text { oxygen }\end{array}$ \\
\hline & & & & & & & & & & & & & $\begin{array}{l}\text { dark } \\
\text { zone }\end{array}$ & $\begin{array}{c}\text { bright } \\
\text { zone }\end{array}$ & & & & Remarks \\
\hline
\end{tabular}

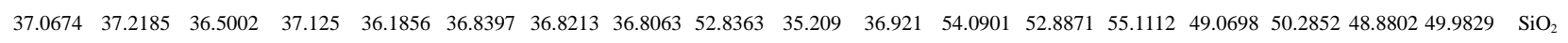

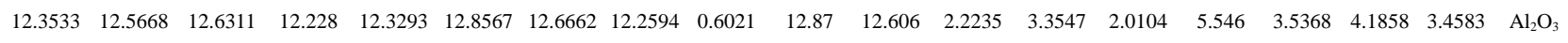

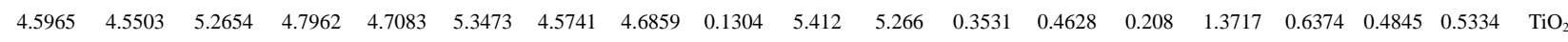
$\begin{array}{lllllllllllllllllllll}0.037 & 0 & 0.0302 & 0 & 0.0033 & 0 & 0 & 0.03 & 0 & 0 & 0 & 0.0298 & 0 & 0 & 0 & 0 & 0.0103 & 0.0338 & \mathrm{Cr}_{2} \mathrm{O}_{3}\end{array}$ $\mathrm{Fe}_{2} \mathrm{O}_{3}$

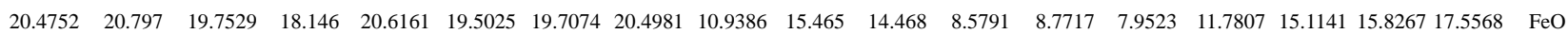

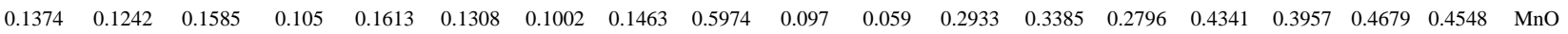

$\mathrm{NiO}$

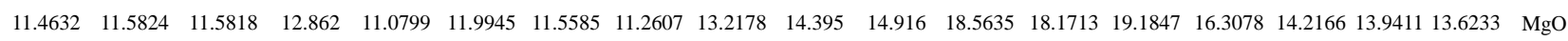

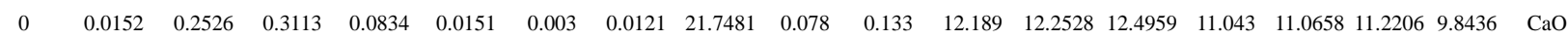

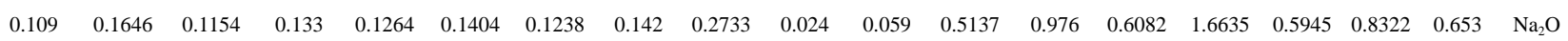

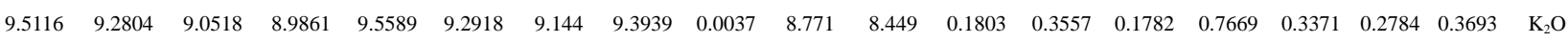

\begin{tabular}{|c|c|c|c|c|c|c|c|c|c|c|c|c|c|c|c|c|c|c|}
\hline 0 & 0 & 0 & 0 & 0 & 0 & 0 & 0 & 0 & 0.034 & 0.032 & 0 & 0 & 0 & 0 & 0 & 0 & 0 & $\mathrm{ZnO}$ \\
\hline 95.751 & 96.299 & 95.340 & 94.692 & 94.853 & 96.119 & 94.699 & 95.235 & 100.348 & 92.355 & 92.909 & 97.015 & 97.571 & 98.029 & 97.984 & 96.183 & 96.128 & 96.509 & Total \\
\hline 5.687 & 5.673 & 5.602 & 5.684 & 5.629 & 5.597 & 5.679 & 5.681 & 7.273 & 5.475 & 5.640 & 7.707 & 7.537 & 7.746 & 7.123 & 7.482 & 7.334 & 7.480 & $\mathrm{Si}$ \\
\hline 2.233 & 2.257 & 2.285 & 2.206 & 2.260 & 2.302 & 2.302 & 2.230 & 0.098 & 2.358 & 2.269 & 0.373 & 0.563 & 0.333 & 0.949 & 0.620 & 0.740 & 0.610 & $\mathrm{Al}$ \\
\hline 0.530 & 0.521 & 0.608 & 0.552 & 0.551 & 0.611 & 0.530 & 0.544 & 0.013 & 0.633 & 0.605 & 0.038 & 0.050 & 0.022 & 0.150 & 0.071 & 0.055 & 0.060 & $\mathrm{Ti}$ \\
\hline 0.004 & 0.000 & 0.004 & 0.000 & 0.000 & 0.000 & 0.000 & 0.004 & 0.000 & 0.000 & 0.000 & 0.003 & 0.000 & 0.000 & 0.000 & 0.000 & 0.001 & 0.004 & $\mathrm{Cr}$ \\
\hline 0.000 & 0.000 & 0.000 & 0.000 & 0.000 & 0.000 & 0.000 & 0.000 & 0.000 & 0.000 & 0.000 & 0.000 & 0.000 & 0.000 & 0.000 & 0.000 & 0.000 & 0.000 & $\mathrm{Fe}^{3+}$ \\
\hline 2.626 & 2.650 & 2.535 & 2.323 & 2.681 & 2.477 & 2.541 & 2.645 & 1.259 & 2.010 & 1.848 & 1.022 & 1.045 & 0.934 & 1.430 & 1.880 & 1.985 & 2.197 & $\mathrm{Fe}^{2+}$ \\
\hline 0.018 & 0.016 & 0.021 & 0.014 & 0.021 & 0.017 & 0.013 & 0.019 & 0.070 & 0.013 & 0.008 & 0.035 & 0.041 & 0.033 & 0.053 & 0.050 & 0.059 & 0.058 & $\mathrm{Mn}$ \\
\hline 0.000 & 0.000 & 0.000 & 0.000 & 0.000 & 0.000 & 0.000 & 0.000 & 0.000 & 0.000 & 0.000 & 0.000 & 0.000 & 0.000 & 0.000 & 0.000 & 0.000 & 0.000 & $\mathrm{Ni}$ \\
\hline 2.620 & 2.630 & 2.648 & 2.933 & 2.567 & 2.715 & 2.656 & 2.589 & 2.710 & 3.334 & 3.394 & 3.940 & 3.858 & 4.017 & 3.526 & 3.151 & 3.116 & 3.037 & $\mathrm{Mg}$ \\
\hline 0.000 & 0.002 & 0.042 & 0.051 & 0.014 & 0.002 & 0.000 & 0.002 & 3.206 & 0.013 & 0.022 & 1.860 & 1.870 & 1.881 & 1.717 & 1.763 & 1.803 & 1.578 & Ca \\
\hline 0.032 & 0.049 & 0.034 & 0.039 & 0.038 & 0.041 & 0.037 & 0.042 & 0.073 & 0.007 & 0.017 & 0.142 & 0.270 & 0.166 & 0.468 & 0.171 & 0.242 & 0.189 & $\mathrm{Na}$ \\
\hline 1.861 & 1.804 & 1.772 & 1.754 & 1.896 & 1.800 & 1.798 & 1.849 & 0.001 & 1.739 & 1.646 & 0.033 & 0.065 & 0.032 & 0.142 & 0.064 & 0.053 & 0.070 & $\mathrm{~K}$ \\
\hline 0.000 & 0.000 & 0.000 & 0.000 & 0.000 & 0.000 & 0.000 & 0.000 & 0.000 & 0.004 & 0.004 & 0.000 & 0.000 & 0.000 & 0.000 & 0.000 & 0.000 & 0.000 & $\mathrm{Zn}$ \\
\hline 15.611 & 15.603 & 15.549 & 15.557 & 15.658 & 15.562 & 15.557 & 15.604 & 14.702 & 15.587 & 15.452 & 15.154 & 15.298 & 15.164 & 15.558 & 15.254 & 15.388 & 15.283 & Total \\
\hline
\end{tabular}




\section{Continued}

\begin{tabular}{|c|c|c|c|c|c|c|c|c|c|c|c|c|c|c|c|c|c|c|}
\hline SN 11 & SN 11 & SN 11 & SN 11 & SN 11 & SN 10 & SN 10 & SK 62 & SK 62 & SK 62 & SK 62 & SK 62 & SK 62 & SN 10 & SS63 & SS63 & SK 62 & SK 62 & $\begin{array}{c}\text { Sample } \\
\text { No. }\end{array}$ \\
\hline Cpx & $\mathrm{px}$ & $\mathrm{px}$ & $\mathrm{px}$ & $\mathrm{px}$ & $\mathrm{px}$ & $\mathrm{px}$ & $\mathrm{px}$ & $\mathrm{px}$ & Cpx & Cpx & $\mathrm{px}$ & $\mathrm{px}$ & $\mathrm{px}$ & Cpx & Cpx & $\mathrm{Bt}$ & $\mathrm{Bt}$ & Mineral \\
\hline 6 & 6 & 6 & 6 & 6 & 6 & 6 & 6 & 6 & 6 & 6 & 6 & 4 & 6 & 6 & 6 & 22 & 22 & $\begin{array}{c}\text { No. } \\
\text { oxygen }\end{array}$ \\
\hline
\end{tabular}

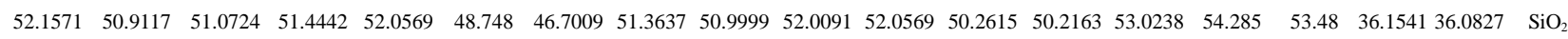

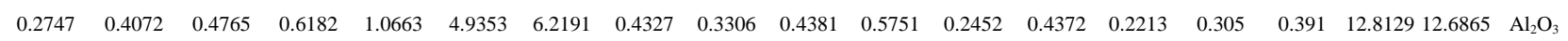

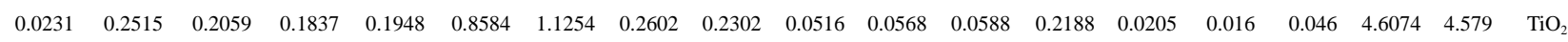
$\begin{array}{llllllllllllllllllllll}0.0316 & 0 & 0.0195 & 0 & 0 & 0 & 0.0085 & 0 & 0.0368 & 0 & 0 & 0 & 0.0032 & 0.0071 & 0.054 & 0 & 0.0067 & 0.005 & \mathrm{Cr}_{2} \mathrm{O}_{3}\end{array}$ $\mathrm{Fe}_{2} \mathrm{O}_{3}$

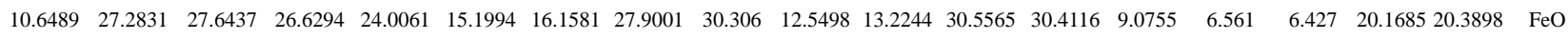

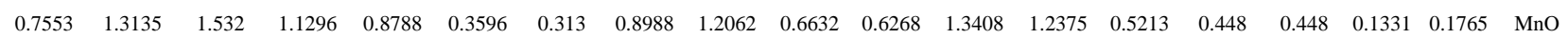

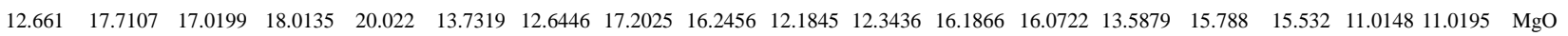

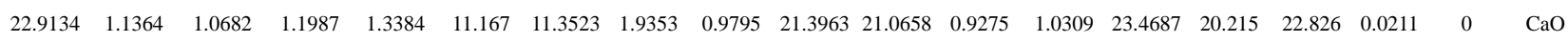

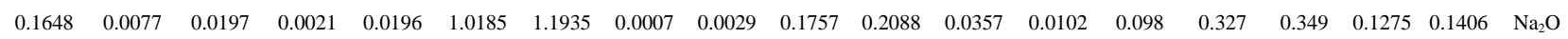
$\begin{array}{lllllllllllllllllllll}0 & 0 & 0.0347 & 0.0091 & 0.0083 & 0.5475 & 0.769 & 0.0026 & 0 & 0 & 0.0037 & 0 & 0 & 0.01 & 0.001 & 0 & 9.4381 & 9.3198 & \mathrm{~K}_{2} \mathrm{O}\end{array}$

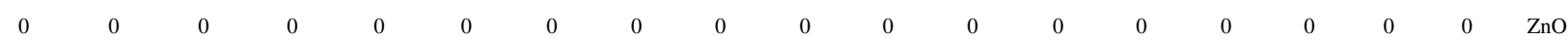

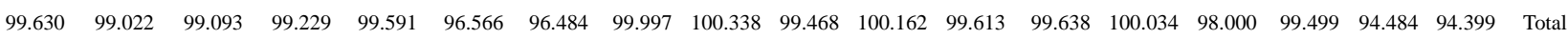

\begin{tabular}{|c|c|c|c|c|c|c|c|c|c|c|c|c|c|c|c|c|c|c|}
\hline 1.981 & 1.978 & 1.986 & 1.984 & 1.972 & 1.896 & 1.837 & 1.980 & 1.980 & 1.986 & 1.977 & 1.973 & 1.312 & 1.989 & 2.029 & 1.988 & 5.624 & 5.623 & $\mathrm{Si}$ \\
\hline 0.012 & 0.019 & 0.022 & 0.028 & 0.048 & 0.226 & 0.288 & 0.020 & 0.015 & 0.020 & 0.026 & 0.011 & 0.013 & 0.010 & 0.013 & 0.017 & 2.348 & 2.330 & $\mathrm{Al}$ \\
\hline 0.001 & 0.007 & 0.006 & 0.005 & 0.006 & 0.025 & 0.033 & 0.008 & 0.007 & 0.001 & 0.002 & 0.002 & 0.004 & 0.001 & 0.000 & 0.001 & 0.539 & 0.536 & $\mathrm{Ti}$ \\
\hline 0.001 & 0.000 & 0.001 & 0.000 & 0.000 & 0.000 & 0.000 & 0.000 & 0.001 & 0.000 & 0.000 & 0.000 & 0.000 & 0.000 & 0.002 & 0.000 & 0.001 & 0.001 & $\mathrm{Cr}$ \\
\hline 0.000 & 0.000 & 0.000 & 0.000 & 0.000 & 0.000 & 0.000 & 0.000 & 0.000 & 0.000 & 0.000 & 0.000 & 0.000 & 0.000 & 0.000 & 0.000 & 0.000 & 0.000 & $\mathrm{Fe}^{3+}$ \\
\hline 0.338 & 0.886 & 0.899 & 0.858 & 0.760 & 0.494 & 0.531 & 0.899 & 0.983 & 0.401 & 0.420 & 1.003 & 0.664 & 0.285 & 0.205 & 0.200 & 2.623 & 2.657 & $\mathrm{Fe}^{2+}$ \\
\hline 0.024 & 0.043 & 0.050 & 0.037 & 0.028 & 0.012 & 0.010 & 0.029 & 0.040 & 0.021 & 0.020 & 0.045 & 0.027 & 0.017 & 0.014 & 0.014 & 0.018 & 0.023 & $\mathrm{Mn}$ \\
\hline 0.000 & 0.000 & 0.000 & 0.000 & 0.000 & 0.000 & 0.000 & 0.000 & 0.000 & 0.000 & 0.000 & 0.000 & 0.000 & 0.000 & 0.000 & 0.000 & 0.000 & 0.000 & $\mathrm{Ni}$ \\
\hline 0.716 & 1.025 & 0.986 & 1.035 & 1.130 & 0.796 & 0.741 & 0.988 & 0.939 & 0.693 & 0.698 & 0.946 & 0.626 & 0.759 & 0.879 & 0.860 & 2.552 & 2.558 & $\mathrm{Mg}$ \\
\hline 0.932 & 0.047 & 0.044 & 0.050 & 0.054 & 0.465 & 0.478 & 0.080 & 0.041 & 0.875 & 0.857 & 0.039 & 0.029 & 0.943 & 0.809 & 0.909 & 0.004 & 0.000 & $\mathrm{Ca}$ \\
\hline 0.012 & 0.001 & 0.001 & 0.000 & 0.001 & 0.077 & 0.091 & 0.000 & 0.000 & 0.013 & 0.015 & 0.003 & 0.001 & 0.007 & 0.024 & 0.025 & 0.038 & 0.042 & $\mathrm{Na}$ \\
\hline 0.000 & 0.000 & 0.002 & 0.000 & 0.000 & 0.027 & 0.039 & 0.000 & 0.000 & 0.000 & 0.000 & 0.000 & 0.000 & 0.000 & 0.000 & 0.000 & 1.872 & 1.852 & $\mathrm{~K}$ \\
\hline 0.000 & 0.000 & 0.000 & 0.000 & 0.000 & 0.000 & 0.000 & 0.000 & 0.000 & 0.000 & 0.000 & 0.000 & 0.000 & 0.000 & 0.000 & 0.000 & 0.000 & 0.000 & $\mathrm{Zn}$ \\
\hline 4.018 & 4.006 & 3.998 & 3.997 & 4.000 & 4.018 & 4.050 & 4.003 & 4.006 & 4.010 & 4.016 & 4.021 & 2.677 & 4.010 & 3.975 & 4.015 & 15.618 & 15.622 & Total \\
\hline
\end{tabular}


Chemical formulae and mineral name for clinopyroxene were done following the method of [21]. In this method, it must be distinguished different kind of clinopyroxenes include sodic, sodiccalcic and calcic, at first step by using Q-J diagram. Then, Wo-En-Fs triangular diagram must be used for determining calcic clinopyroxenes and Q-Jd-Ae triangular diagram for classifying sodic and sodiccalcic clinopyroxenes.

For amphiboles, Chemical formulae and mineral name distinguished by using the [22] method. In this method, based on occupancy of B location in the mineral structure by $\mathrm{Ca}$ and $\mathrm{Na}, 4$ main groups of amphiboles (rich in Fe-Mg-Mn, calcic, sodic-calcic and sodic groups) were recognized. Then, exact name and composition of amphibole identified by using $\mathrm{Mg} /\left(\mathrm{Mg}+\mathrm{Fe}^{2}\right)$ vs. Si binary diagrams.

Chemical composition and classifying of biotite were done by using $\mathrm{Fe} /(\mathrm{Fe}+\mathrm{Mg}) \mathrm{vs}$. $\mathrm{Al}^{\mathrm{IV}}$ binary diagram. Finally, feldspars (include plagioclases and alkali feldspars) classified by using the Or-Ab-An triangular diagram.

As mentioned in the petrography section, clinopyroxenes present in the all phases of Neshveh intrusion, but it is predominant at the quartz monzogabbros. All of the analyzed clinopyroxenes classified as calcic clinopyroxenes (Figure 6(A)). Most of the clinopyroxenes in the quartz monzogabbros are clinoenstatite-clinofferosillite but some minerals have diopside and augite composition (Figure 6(B)). Clinopyroxenes within the quartz monzodiorites and granites have diopside and augite-diopside composition (Figure 6(B)).

As mentioned in the petrography section, clinopyroxenes present in the all phases of Neshveh intrusion, but it is predominant at the quartz monzogabbros. All of the analyzed clinopyroxene classified as calcic clinopyroxenes (Figure 6(A)). Most of the clinopyroxenes in the quartz monzogabbros are clinoenstatite-clinofferosillite but some minerals have diopside and augite composition (Figure 6(B)). Clinopyroxenes within the quartz monzodiorites and granites have diopside and augite-diopside composition (Figure 6(B)).

All of the analyzed amphiboles from the different parts of the Neshveh intrusion are classified as calcic amphiboles (Figure 7(A)). Based on $\mathrm{Mg} /\left(\mathrm{Mg}+\mathrm{Fe}^{2}\right)$ vs. Si binary diagrams, amphiboles of the quartz monzogabbros have actinolitic hornblende composition, analyzed amphiboles from the quartz monzodiorites are hornblende, Fe-hornblende, Mg-hornblende and actinolite, amphiboles from the granodiorites are Mg-hornblende and finally, amphiboles of the granites have actinolitic composition (Figure 7(B)). Most of them are edenite in composition (Figure 7(C)).

Based on $\mathrm{Fe} /(\mathrm{Fe}+\mathrm{Mg})$ vs. $\mathrm{Al}^{\mathrm{IV}}$ binary diagram, analyzed biotites from the quartz monzogabbros and quartz monzodiorites have intermediate composition between the phlogopite and annite (Figure 8(A)).

Plagioclase is the most common mineral that occur in all phases of the Neshveh intrusion. Its composition varies within the quartz monzogabbros, quartz monzodiorites, granodiorites and granites. As mentioned before, within the granitic and granodioritic parts of the Neshveh intrusion, there are some plagioclases that their compositions vary at the core and rims (Figure 5(B)). This subject is supported by EPMA analysis, also. Analyzed
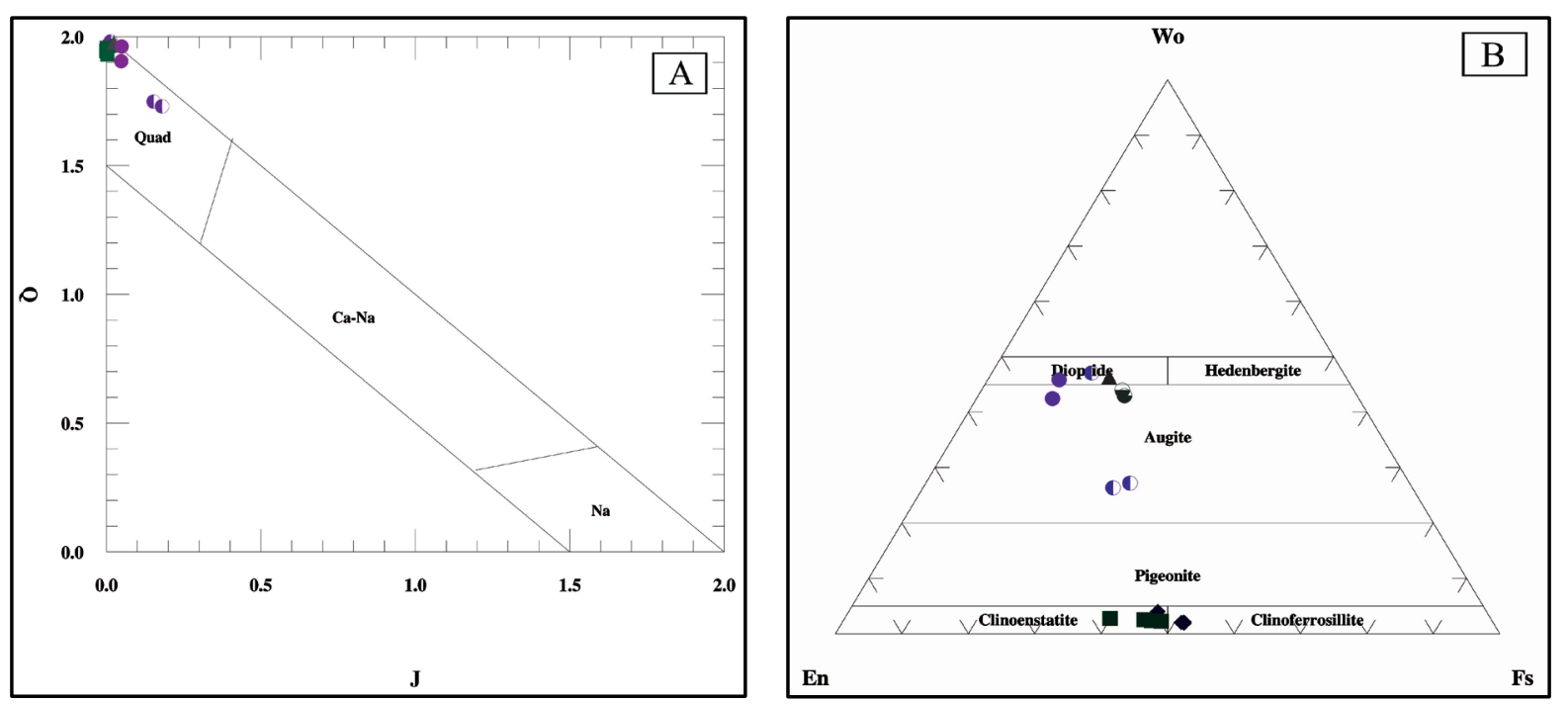

Figure 6. (A) Location of the analyzed clinopyroxenes on the Q-J diagram of Morimoto (1988); (B) Classifying of the analyzed clinopyroxenes based on Wo-En-Fs triangular diagram [21]. 

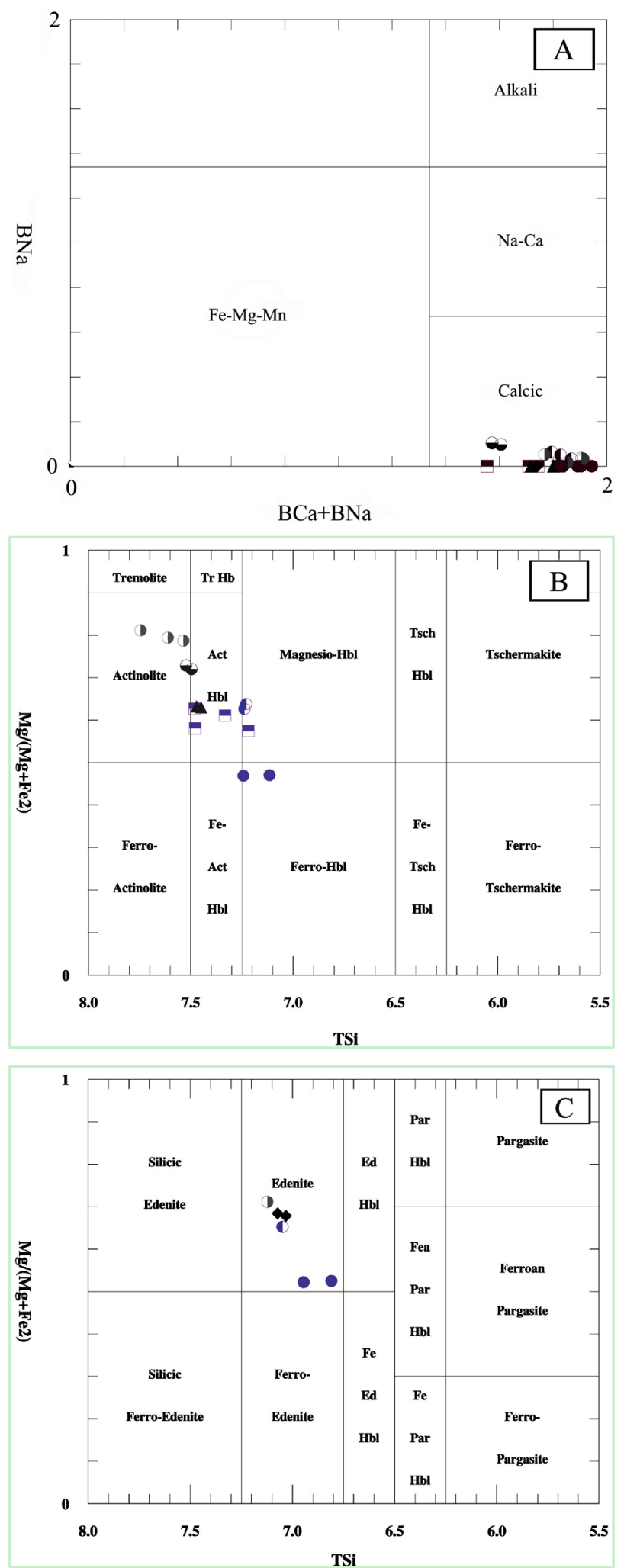

Figure 7. (A) Classification of the analyzed amphiboles based on occupancy of B location in the mineral structure by $\mathrm{Ca}$ and $\mathrm{Na}$ [22]; (B) and (C) Classifying of the analyzed amphiboles based on $\mathrm{Mg} /\left(\mathrm{Mg}+\mathrm{Fe}^{2}\right) \mathrm{vs}$. Si binary diagram [22]. 

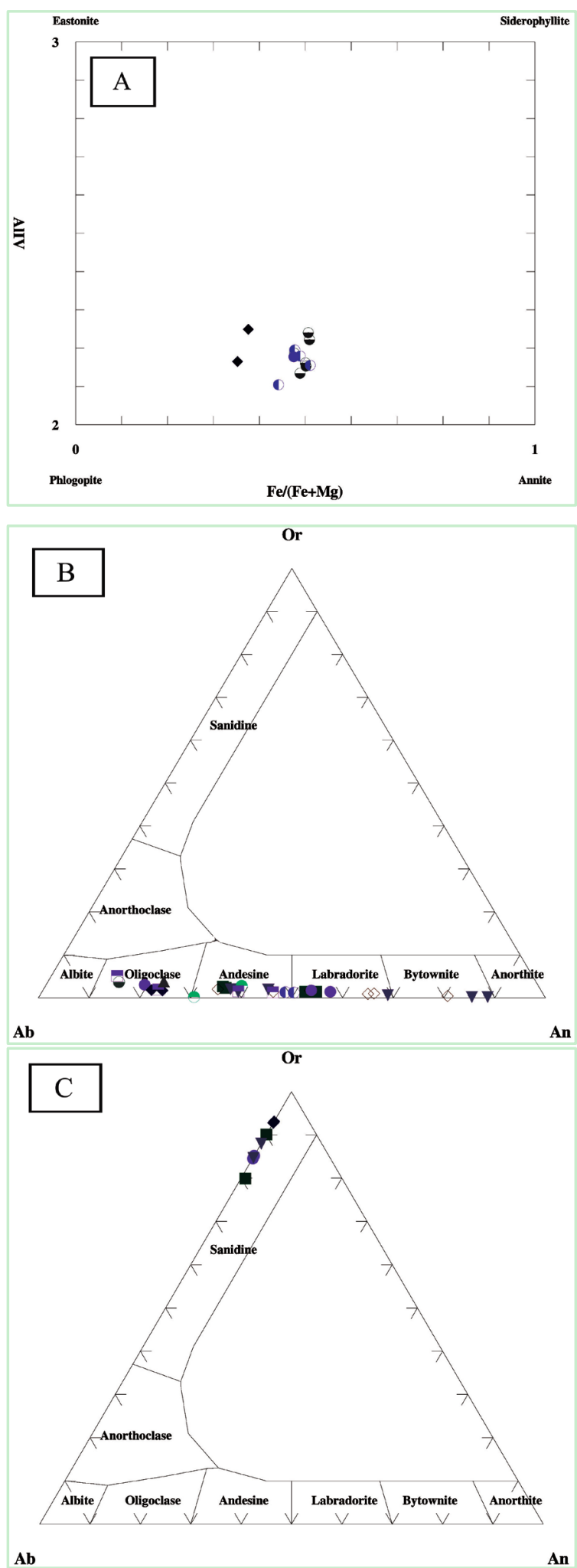

Figure 8. (A) Classification of the analyzed biotites based on $\mathrm{Fe} /(\mathrm{Fe}+$ $\mathrm{Mg})$ vs. $\mathrm{Al}^{\mathrm{IV}}$ binary diagram; (B) Classification of the analyzed plagioclases based on the Or-Ab-An triangular diagram; (C) Classification of the analyzed alkali feldspars based on the Or-Ab-An triangular diagram. 
plagioclases from the quartz monzogabbros indicate that most of them are labradorite and some bytownite (Figure 8(B)). The rims of some crystals are andesine in composition (Figure 8(B)). Plagioclases from the quartz monzodiorite are mainly andesine and occasionally labradorite (Figure 8(B)). Some of them indicate oligoclase composition and in one point, the core of plagioclase has bytownite composition (Figure 8(B)). Plagioclases of the granodiorites have oligoclase compositionbut core of some crystals show different and calcic composition which is labradorite and andesine (Figure 8(B)). Plagioclases in the granites also have oligoclase composition but some crystals have calcic core that its composition is andesine (Figure 8(B)).

All analyzed alkali feldspars locate near the Or corner on the Or-Ab-An triangular diagram (Figure 8(C)).

\section{Geochemistry}

As mentioned before, 15 fresh whole-rock samples representative of the petrological range in the Neshveh intrusion were analyzed for major and trace elements and REEs by ICP and ICP-MS method at ALS Chemex laboratory in Canada (Table 3).

The calc-alkaline nature of the all rock types of the Neshveh intrusion is illustrated on the $\mathrm{SiO}_{2} \mathrm{vs} \mathrm{K}_{2} \mathrm{O}$ diagram [23], which are the samples all plot within the medium to high-K calcalkaline field (Figure 9). The molecular ratio of $\mathrm{Al}_{2} \mathrm{O}_{3} /\left(\mathrm{CaO}+\mathrm{Na}_{2} \mathrm{O}+\mathrm{K}_{2} \mathrm{O}\right)$ ranges from 0.73 to 1 , exhibiting metaluminous characteristics [15]. Features such as $\mathrm{Na}_{2} \mathrm{O}>\mathrm{K}_{2} \mathrm{O}$, A/CNK $<1$, decreasing $\mathrm{P}_{2} \mathrm{O}_{5}$ versus $\mathrm{SiO}_{2}$ [24] and $\mathrm{SiO}_{2} \mathrm{vs.}_{2} \mathrm{O}$ [25] and ACNK versus $\mathrm{SiO}_{2}$ diagrams [26] are characteristic of I-type rocks, which is also consistent with the presence of key modal minerals such as hornblende and titaniteand absence of muscovite, garnet, cordierite, andalusite and sillimanite. The rocks are sodic as shown by the high average values of $\mathrm{Na}_{2} \mathrm{O} / \mathrm{K}_{2} \mathrm{O}(1.53)$ and $\mathrm{Na}_{2} \mathrm{O}+\mathrm{K}_{2} \mathrm{O}(5.33)$ (Table 3).

The Neshveh intrusion rocks have a wide range in $\mathrm{SiO}_{2}(52.1 \%-71.2 \%), \mathrm{Fe}_{2} \mathrm{O}_{3}, \mathrm{MgO}, \mathrm{MnO}, \mathrm{CaO}, \mathrm{TiO}_{2}$ and $\mathrm{P}_{2} \mathrm{O}_{5}$ (Table 3). Most major elements except $\mathrm{Al}_{2} \mathrm{O}_{3}, \mathrm{Na}_{2} \mathrm{O}$ and $\mathrm{K}_{2} \mathrm{O}$ show negative linear trends with increasing $\mathrm{SiO}_{2}$ (Figure 10). $\mathrm{K}_{2} \mathrm{O}$ shows a descending trend, while $\mathrm{Al}_{2} \mathrm{O}_{3}$ and $\mathrm{Na}_{2} \mathrm{O}$ have bent trends. $\mathrm{Na}_{2} \mathrm{O}$ is positive up to $62 \mathrm{wt} \% \mathrm{SiO}_{2}$ and negative from this point onward.

In the Harker diagrams (Figure 11), Ba, Rb, Zr, Nb, and Ce show ascending linear trends, whereas $\mathrm{V}$ and $\mathrm{Co}$ decrease with increasing silica content. Sr, Eu and Y follow curved trends that suggest these elements behaved incompatibly in the magmas that formed the quartz monzogabbro and quartz monzodiorite, and compatibly during the crystallization of granodiorite and granite.

The samples display similar chondrite-normalized REE patterns. They are characterized by LREE enrichment with $(\mathrm{La} / \mathrm{Sm})_{\mathrm{N}}=2.11-4.27$ and weakly fractionated HREE with $(\mathrm{Gd} / \mathrm{Yb})_{\mathrm{N}}=1.16-1.46$, suggesting garnet-free sources [27]. The mafic to intermediate rocks have slight but very consistent negative Eu anomalies $\left(\mathrm{Eu} / \mathrm{Eu}^{*}=\right.$ 0.91 - 0.82), decreasing more markedly in the granodiorites to a minimum of 0.42 in the granite with 71 wt\% $\mathrm{SiO}_{2}$ (Figure 12(A)).The primitive mantle normalized spider diagrams for different phases of Neshveh intrusion demonstrate negative anomalies of HFSEs including $\mathrm{Nb}$, $\mathrm{Ti}$ and $\mathrm{P}$, along with positive anomalies in LILEs including $\mathrm{Rb}, \mathrm{Ba}, \mathrm{Th}, \mathrm{K}$ and $\mathrm{Pb}$ (Figure 12(B)).

These geochemical characteristics, with light-REE enrichment, positive $\mathrm{Pb}$ anomaly and the Nb-Ti troughs on the spider diagram are typical of calc alkaline magmatism in active continental margins [29]. A marked Nb-Ta trough in primitive-mantle normalized trace element patterns has been ascribed to retention of these elements in mineral phases containing $\mathrm{Ti}$ (e.g., rutile, ilmenite, rutile, garnet and some amphiboles) during dehydration of subducted oceanic crust or crustal contamination [30]. With increasing pressure, the solubility of titanium bearing minerals reduces in aqueous fluids [31]. Therefore, theses minerals that are rich in HFSEs will be stable during partial melting process at depths greater than $30 \mathrm{~km}$ that is led to negative anomalies in the melt [31]. The negative anomaly in phosphorus indicative for low content of apatite is in the studied rocks. As mentioned before, in the I-type granitoids, phosphorus acts as a consistent element and fractionation this element in the early stages of magma crystallization, led to negative anomalies in the crystalized rocks.

Fluids and melts derived from the subducted oceanic slab, led to the metasomatism of upper mantle wedge and thereupon, negative anomalies of $\mathrm{Nb}$ and $\mathrm{Ta}$ [27] [32] [33]. Positive anomalies in $\mathrm{Pb}, \mathrm{K}, \mathrm{Rb}$ and $\mathrm{Ba}$ are attributed to metasomatism of mantle wedge by fluids derived from the subducted slab or/ and contamination with continental crust [34] [35].

The Chondrite-normalized REE patterns in the different phases of the Neshveh intrusion indicate negative Eu anomalies (Figure 12(A)). Negative anomalies in Eu are related to the feldspar fractionation during magma crystallization or remaining of feldspar at the source. If these negative anomalies are associated with negative 
Table 3. Major (wt. \%) and trace element (ppm) abundances in Neshveh intrusion samples.

\begin{tabular}{|c|c|c|c|c|c|c|c|c|c|c|c|c|c|c|c|}
\hline \multirow[b]{2}{*}{ Sample No. } & \multicolumn{6}{|c|}{ Quartz monzogabbro } & \multicolumn{4}{|c|}{ Quartz monzodiorite } & \multicolumn{3}{|c|}{ Granodiorite } & \multicolumn{2}{|c|}{$\underline{\text { Granite }}$} \\
\hline & SK11 & SK42 & SK18 & SK5 & SK64 & SK58 & SN10 & SK62 & SN11 & SK66 & SN17 & SN15 & SN44 & SN52 & SK56 \\
\hline $\mathrm{SiO}_{2}$ & 52.1 & 52.2 & 53.2 & 54.7 & 55.5 & 55.1 & 56.5 & 56.7 & 59.6 & 60.8 & 62.5 & 62.7 & 65.1 & 69.4 & 71.2 \\
\hline $\mathrm{TiO}_{2}$ & 0.93 & 1.01 & 0.82 & 0.87 & 0.8 & 0.74 & 0.73 & 0.75 & 0.71 & 0.71 & 0.51 & 0.57 & 0.49 & 0.32 & 0.33 \\
\hline $\mathbf{A l}_{2} \mathbf{O}_{3}$ & 17.05 & 14.9 & 16.25 & 17.85 & 16.45 & 17.95 & 17.1 & 16.55 & 16.7 & 15.7 & 15.45 & 16.15 & 14.8 & 13.8 & 12.7 \\
\hline $\mathrm{Fe}_{2} \mathrm{O}_{3}$ & 11.1 & 12.85 & 10.3 & 8.96 & 8.59 & 8.95 & 8.66 & 9.38 & 7.66 & 7.66 & 5.07 & 6.03 & 3.89 & 3.05 & 3.05 \\
\hline MnO & 0.19 & 0.29 & 0.3 & 0.22 & 0.12 & 0.11 & 0.14 & 0.2 & 0.14 & 0.14 & 0.13 & 0.15 & 0.05 & 0.07 & 0.05 \\
\hline MgO & 4.08 & 4.11 & 4.43 & 2.32 & 3.75 & 3.1 & 3.26 & 3.6 & 2.48 & 2.41 & 2.05 & 1.78 & 1.52 & 1.1 & 0.6 \\
\hline $\mathrm{CaO}$ & 7.44 & 7.71 & 6.33 & 6.83 & 7.66 & 7.06 & 7.16 & 7.71 & 5.89 & 5.19 & 3.38 & 4.81 & 4.84 & 2.27 & 1.58 \\
\hline $\mathrm{Na}_{2} \mathrm{O}$ & 3.39 & 2.42 & 3.1 & 4.09 & 3.82 & 3.68 & 3.23 & 3.14 & 3.59 & 3.44 & 3.64 & 3.49 & 3.47 & 3.06 & 2.56 \\
\hline $\mathrm{K}_{2} \mathrm{O}$ & 1.48 & 1.88 & 2.1 & 1.62 & 1.21 & 1.93 & 1.85 & 1.76 & 2.31 & 2.38 & 3.46 & 2.73 & 2.9 & 4.19 & 5.14 \\
\hline $\mathbf{P}_{2} \mathbf{O}_{5}$ & 0.23 & 0.26 & 0.15 & 0.43 & 0.25 & 0.26 & 0.23 & 0.22 & 0.2 & 0.22 & 0.15 & 0.19 & 0.15 & 0.09 & 0.11 \\
\hline Total & 98 & 97.6 & 97 & 97.9 & 98.2 & 98.9 & 98.9 & 100 & 99.3 & 98.7 & 96.3 & 98.6 & 97.2 & 97.4 & 97.3 \\
\hline $\mathbf{V}$ & 353 & 426 & 338 & 208 & 295 & 226 & 241 & 269 & 194 & 188 & 89 & 128 & 76 & 47 & 52 \\
\hline $\mathrm{Cr}$ & 30 & 10 & 20 & 10 & 30 & 10 & 20 & 30 & 10 & 10 & 10 & 10 & 10 & 10 & 10 \\
\hline $\mathrm{Ni}$ & 16 & 13 & 14 & 6 & 13 & 7 & 14 & 11 & 8 & 8 & 5 & 8 & 5 & 5 & 6 \\
\hline Co & 30.3 & 32.4 & 29.8 & 15.8 & 17.7 & 18.5 & 3.8 & 24.4 & 17.4 & 16.6 & 8 & 12.8 & 5 & 4 & 5.5 \\
\hline $\mathrm{Cu}$ & 264 & 121 & 451 & 36 & 33 & 49 & 53 & 52 & 48 & 29 & 22 & 47 & 5 & 8 & 25 \\
\hline Zn & 104 & 120 & 169 & 66 & 49 & 45 & 51 & 92 & 75 & 65 & 80 & 76 & 23 & 44 & 40 \\
\hline Ga & 17.9 & 16.9 & 17.3 & 18 & 17.1 & 18.1 & 17.5 & 16.7 & 17.7 & 16.3 & 15 & 16.2 & 14.4 & 13.2 & 12.9 \\
\hline Ba & 341 & 422 & 554 & 361 & 247 & 495 & 465 & 428 & 548 & 605 & 701 & 675 & 759 & 808 & 500 \\
\hline Sr & 391 & 336 & 369 & 403 & 347 & 589 & 503 & 383 & 424 & 394 & 347 & 449 & 353 & 248 & 146 \\
\hline $\mathbf{R b}$ & 33.1 & 38.6 & 46.1 & 40.4 & 27.9 & 40.4 & 48.7 & 43.3 & 60.9 & 56 & 74.2 & 71.4 & 38.1 & 90.8 & 201 \\
\hline Nb & 4.4 & 5.5 & 4.3 & 6.1 & 4.9 & 4.3 & 4.8 & 4.6 & 6.6 & 6.6 & 7.9 & 6.6 & 7.4 & 8.7 & 9.9 \\
\hline $\mathbf{Y}$ & 22.4 & 25.3 & 21.3 & 27.7 & 23.9 & 21.7 & 20.9 & 25 & 23 & 25.7 & 21.6 & 20.4 & 18.4 & 16.2 & 23.2 \\
\hline $\mathrm{Zr}$ & 81 & 81 & 79 & 121 & 94 & 72 & 83 & 95 & 119 & 145 & 134 & 121 & 145 & 124 & 189 \\
\hline Cs & 1.25 & 0.67 & 0.87 & 0.68 & 0.74 & 1.19 & 1.95 & 1.5 & 2.34 & 1.88 & 1.49 & 1.22 & 0.4 & 1.47 & 3.07 \\
\hline Hf & 2.4 & 2.8 & 2.4 & 3.6 & 2.9 & 2.2 & 2.4 & 2.8 & 3.5 & 4.2 & 3.8 & 3.6 & 4.1 & 3.8 & 6.2 \\
\hline Ta & 0.3 & 0.4 & 0.3 & 0.4 & 0.3 & 0.3 & 0.3 & 0.3 & 0.5 & 0.4 & 0.5 & 0.5 & 0.5 & 0.7 & 0.8 \\
\hline Th & 3.35 & 5.69 & 3.39 & 5.41 & 4.02 & 2.72 & 3.17 & 3.56 & 5.11 & 4.62 & 5.89 & 5.55 & 5.91 & 8.42 & 21.1 \\
\hline $\mathbf{U}$ & 0.99 & 1.38 & 1.02 & 1.64 & 1.15 & 0.73 & 0.71 & 1.02 & 1.23 & 1.47 & 1.3 & 1.43 & 1.56 & 1.78 & 5.18 \\
\hline La & 10.9 & 12.9 & 9.6 & 14.1 & 11.5 & 13.7 & 14 & 11.8 & 15.3 & 16.5 & 16.8 & 24.2 & 16 & 19.7 & 19.9 \\
\hline Ce & 22.3 & 26.4 & 20 & 28.5 & 23.7 & 28.2 & 28.4 & 24.5 & 30.8 & 33.3 & 32.6 & 45.7 & 31.6 & 34.9 & 38.1 \\
\hline Pr & 2.97 & 3.57 & 2.62 & 3.73 & 3.07 & 3.7 & 3.64 & 3.22 & 3.85 & 4.16 & 4.07 & 5.24 & 3.85 & 3.9 & 4.39 \\
\hline Nd & 12.9 & 14.5 & 11.2 & 15.9 & 13.3 & 15.4 & 14.9 & 13.5 & 15.4 & 16.8 & 16.2 & 19.3 & 14.9 & 14.2 & 16.3 \\
\hline Sm & 3.18 & 3.68 & 2.85 & 3.91 & 3.24 & 3.64 & 3.38 & 3.31 & 3.53 & 4.02 & 3.7 & 3.76 & 3.21 & 2.84 & 3.54 \\
\hline
\end{tabular}




\begin{tabular}{|c|c|c|c|c|c|c|c|c|c|c|c|c|c|c|c|}
\hline \multicolumn{16}{|l|}{ Continued } \\
\hline Eu & 0.97 & 1.06 & 0.93 & 1.2 & 0.95 & 1.02 & 0.99 & 1.05 & 0.94 & 1.04 & 0.91 & 0.95 & 0.88 & 0.6 & 0.51 \\
\hline Gd & 3.96 & 4.21 & 3.42 & 4.62 & 3.83 & 3.92 & 3.67 & 4.24 & 3.87 & 4.4 & 4.15 & 3.91 & 3.65 & 2.99 & 3.85 \\
\hline $\mathbf{T b}$ & 0.61 & 0.72 & 0.57 & 0.75 & 0.64 & 0.62 & 0.59 & 0.69 & 0.62 & 0.69 & 0.64 & 0.56 & 0.55 & 0.46 & 0.63 \\
\hline Dy & 4.02 & 4.62 & 3.83 & 4.85 & 4.12 & 3.92 & 3.73 & 4.38 & 4 & 4.44 & 3.88 & 3.48 & 3.26 & 2.8 & 4.03 \\
\hline Ho & 0.83 & 0.94 & 0.81 & 0.98 & 0.87 & 0.75 & 0.77 & 0.93 & 0.8 & 0.94 & 0.83 & 0.71 & 0.69 & 0.6 & 0.85 \\
\hline Er & 2.35 & 2.74 & 2.48 & 2.99 & 2.68 & 2.24 & 2.18 & 2.67 & 2.45 & 2.76 & 2.41 & 2.12 & 2.12 & 1.89 & 2.57 \\
\hline $\mathbf{T m}$ & 0.36 & 0.41 & 0.35 & 0.43 & 0.38 & 0.35 & 0.32 & 0.41 & 0.34 & 0.42 & 0.37 & 0.32 & 0.32 & 0.29 & 0.38 \\
\hline $\mathbf{Y b}$ & 2.35 & 2.56 & 2.34 & 2.65 & 2.47 & 2.18 & 2.24 & 2.62 & 2.39 & 2.68 & 2.44 & 2.14 & 2.16 & 2.06 & 2.65 \\
\hline Lu & 0.37 & 0.42 & 0.36 & 0.43 & 0.39 & 0.38 & 0.33 & 0.45 & 0.4 & 0.47 & 0.37 & 0.36 & 0.33 & 0.34 & 0.44 \\
\hline $\mathbf{P b}$ & 18 & 5 & 75 & 6 & 8 & 6 & 7 & 8 & 11 & 12 & 21 & 13 & 6 & 17 & 11 \\
\hline $\mathrm{Na}_{2} \mathrm{O} / \mathrm{K}_{2} \mathrm{O}$ & 2.29 & 1.29 & 1.48 & 2.52 & 3.16 & 1.91 & 1.75 & 1.78 & 1.55 & 1.45 & 1.05 & 1.28 & 1.2 & 0.73 & 0.5 \\
\hline $\mathrm{Na}_{2} \mathrm{O}+\mathrm{K}_{2} \mathrm{O}$ & 4.87 & 4.3 & 5.2 & 5.71 & 5.03 & 5.61 & 5.08 & 4.9 & 5.9 & 5.82 & 7.1 & 6.22 & 6.37 & 7.25 & 7.7 \\
\hline $\mathbf{R b} / \mathbf{S r}$ & 0.08 & 0.11 & 0.12 & 0.1 & 0.08 & 0.07 & 0.1 & 0.11 & 0.14 & 0.14 & 0.21 & 0.16 & 0.11 & 0.37 & 1.38 \\
\hline $\mathbf{E u} / \mathbf{E u}^{*}$ & 0.84 & 0.83 & 0.91 & 0.87 & 0.83 & 0.82 & 0.86 & 0.86 & 0.78 & 0.76 & 0.71 & 0.75 & 0.79 & 0.63 & 0.42 \\
\hline
\end{tabular}
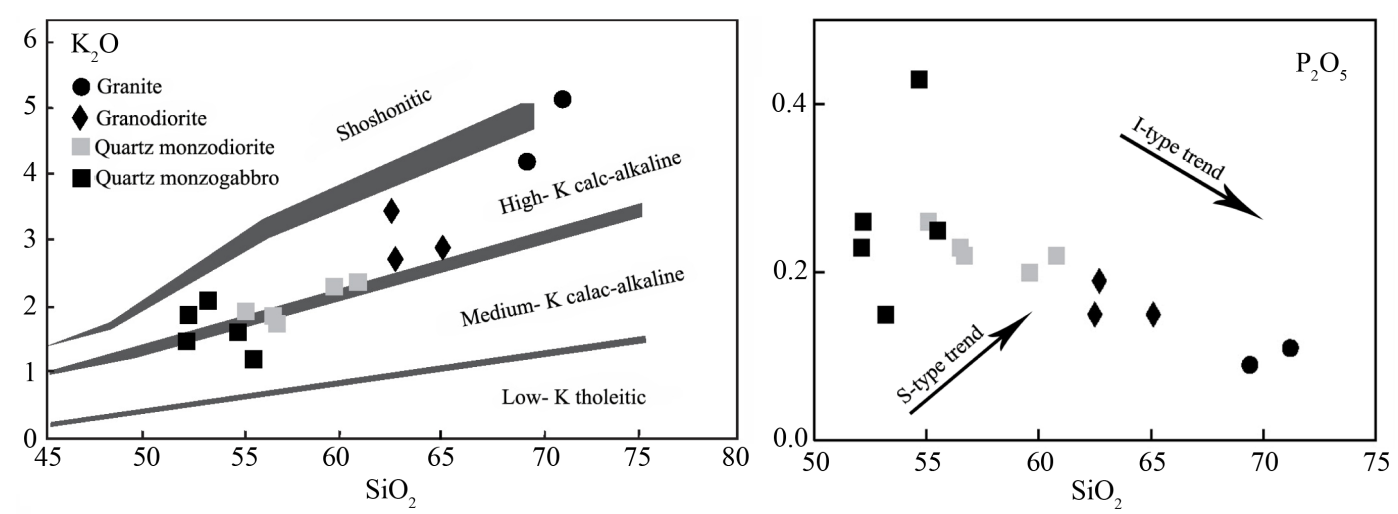

Figure 9. (A) Plot of $\mathrm{K}_{2} \mathrm{O}$ vs. $\mathrm{SiO}_{2}$ showing the calc-alkaline nature for the Neshveh intrusion [23]; (B) Decreasing of $\mathrm{P}_{2} \mathrm{O}_{5}$ vs. $\mathrm{SiO}_{2}$ indicating that Neshveh intrusion is I-type.

anomalies in Sr, fractional crystallization of plagioclase is responsible for these anomalies. While, the negative anomalies in Eu are associated with negative anomalies in Ba, K-feldspar fractionation has the main role in this respect [27]. In the studied rocks, negative anomalies of Eu occur along with Sr, which refers to the plagioclase fractionation.

In terms of geodynamic setting, this intrusion classified as volcanic arc granites (VAG) and active continental margin granites (CAG) [15] [36]. Geochemical studies indicate that the Neshveh intrusion was formed in a volcanic arc and active continental margin. In this base, it is assumed that this intrusion was formed as a result of Neotethys oceanic lithosphere subduction beneath the Central Iran zone in which replaced in the Sahand-Bazman magmatic arc.

\section{Crystal Fractionation Evidences}

Volcanic arc igneous rocks in orogenic belts mostly range in composition from gabbro-diorite to granite [37][41]. This wide variation is variously ascribed to crystal fractionation, multi-pulse intrusion when the new pulse has different composition compare to the previous magma, magma mixing, variable degrees of restite separation, and contamination by assimilation [42] [43]. 

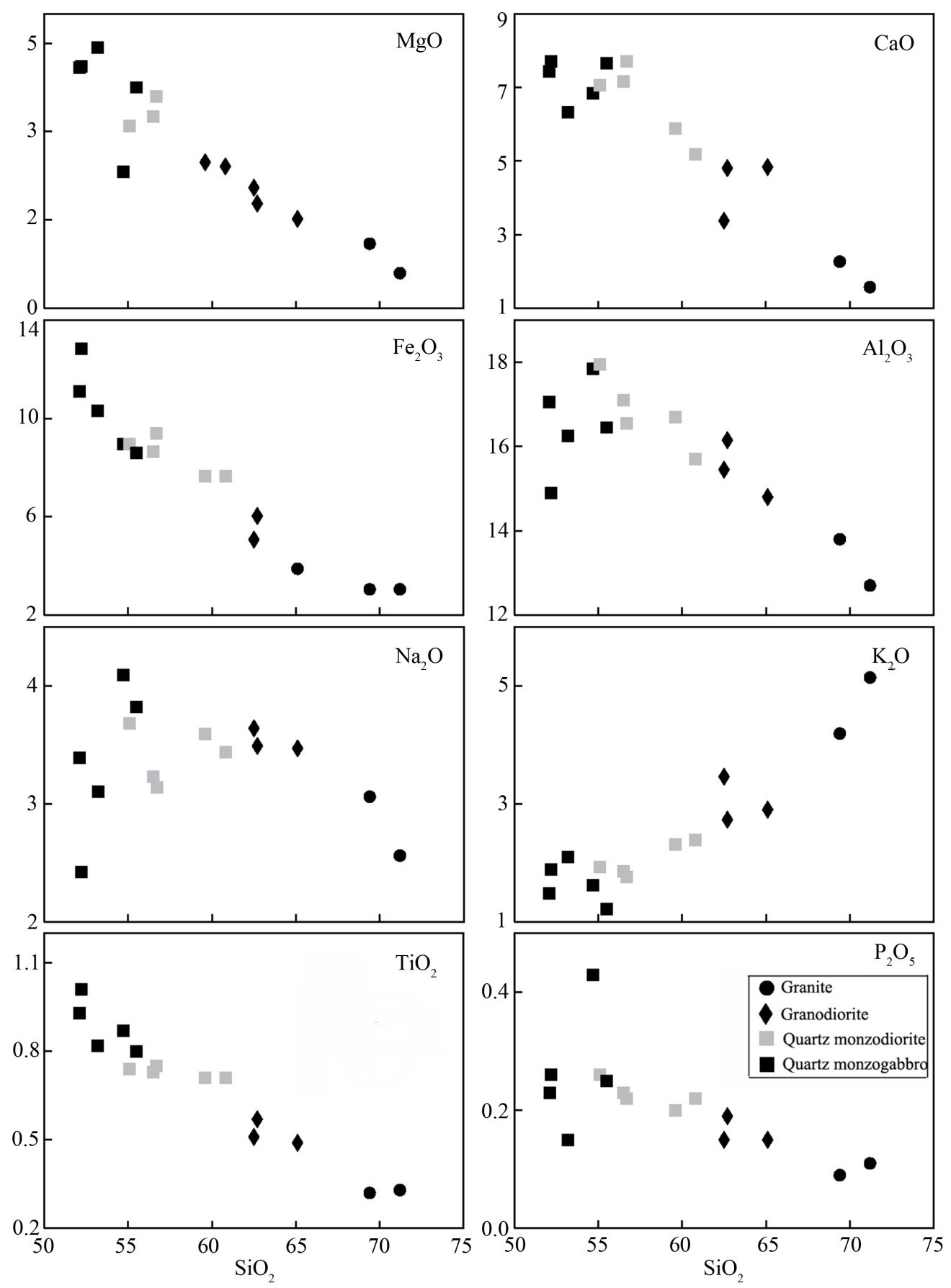

Figure 10. Selected major oxides vs. $\mathrm{SiO}_{2}$ (wt\%) contents for the Neshveh intrusion samples.

The types of change that are typically seen during magmatic differentiation [44] [45] include: (a) major elements such as $\mathrm{SiO}_{2}$ and $\mathrm{K}_{2} \mathrm{O}$ increase in abundance, but others such as $\mathrm{TiO}_{2}, \mathrm{Fe}_{2} \mathrm{O}_{3}$, $\mathrm{MgO}$ and CaO decrease, (b) $\mathrm{Mg} / \mathrm{Fe}$ and $\mathrm{Ca} / \mathrm{Na}$ ratios decrease, (c) concentrations of trace elements such as Rb, Sn, Cs, W and U rise, (d) those elements such as $\mathrm{V}, \mathrm{Cr}, \mathrm{Ni}, \mathrm{Zn}$ and $\mathrm{Sr}$ fall, (e) some trace elements may rise or fall depending on whether or not the melt is saturated in the dominant mineral containing that element, e.g. $\mathrm{Zr}$ and $\mathrm{Ba}$, (f) in some cases mineral saturation in felsic melts depends on whether the melt is I- or S-type, the most important example being apatite saturation which is a feature of felsic I-type melts ( $\mathrm{P}$ falls) but not of more strongly peraluminous S-type melts ( $\mathrm{P}$ rises). 

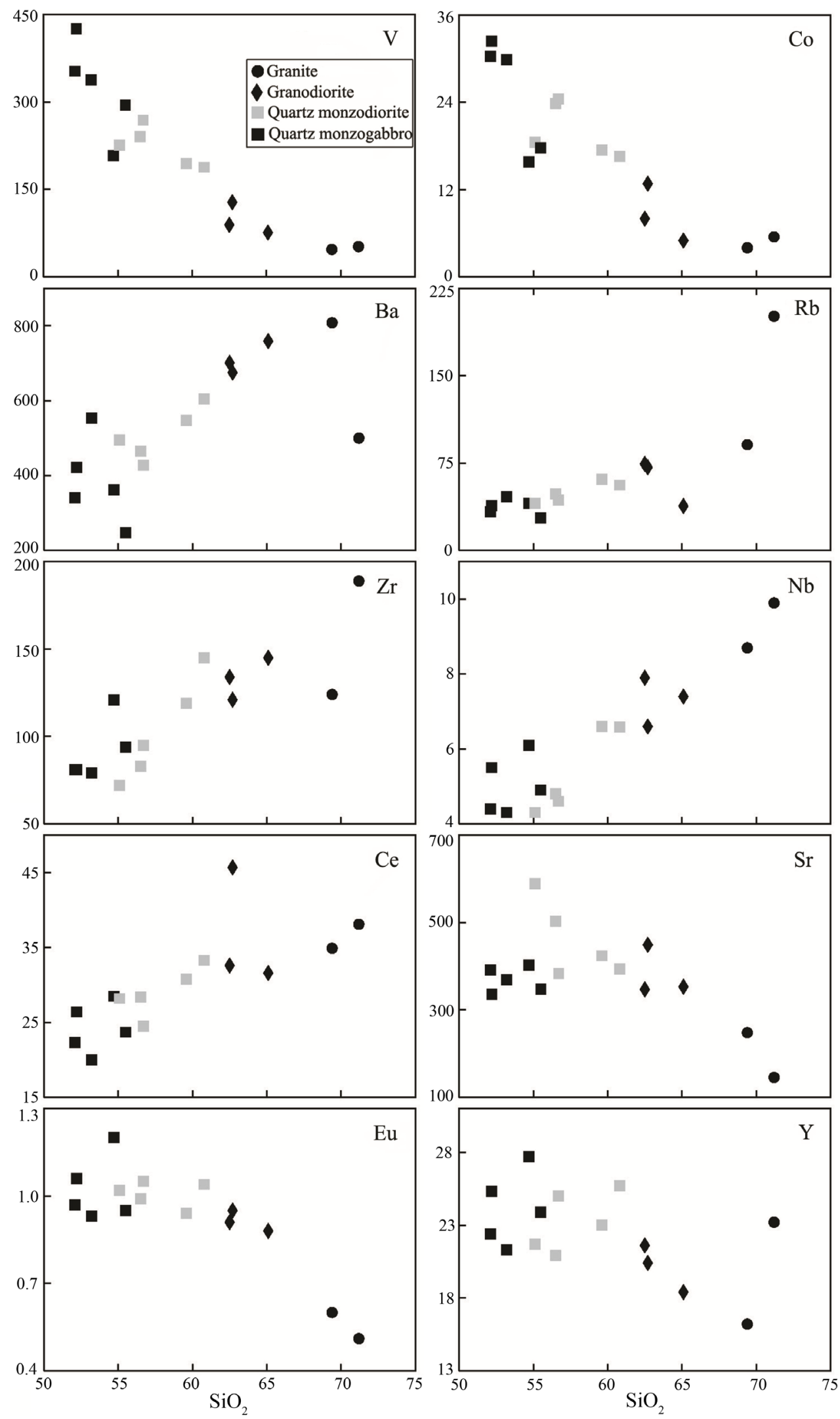

Figure 11. Selected trace elements (ppm) vs. $\mathrm{SiO}_{2}$ (wt\%) contents for the Neshveh intrusion samples. 

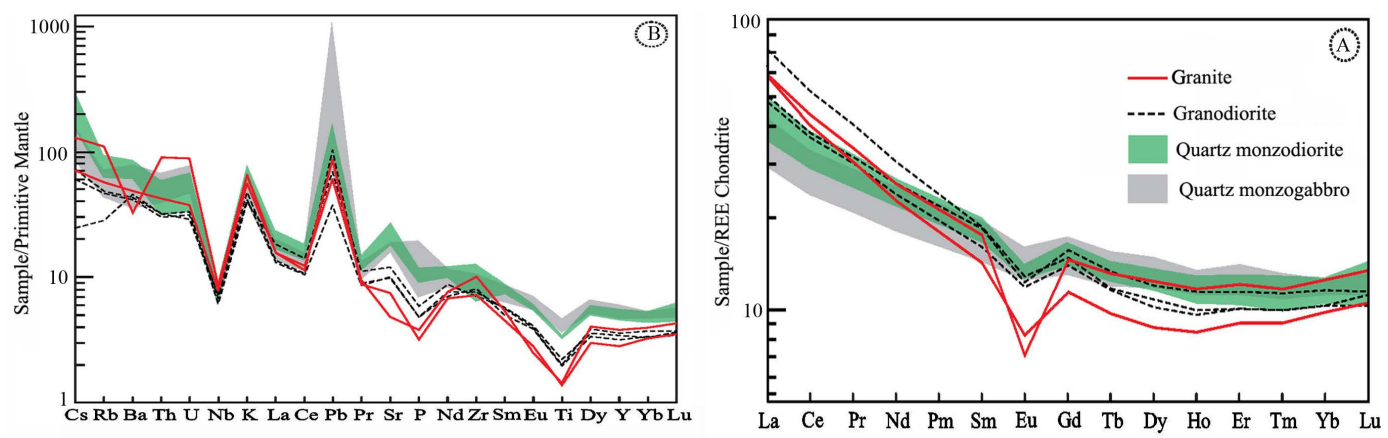

Figure 12. Chondrite-normalized rare earth element plot of the Neshveh intrusion samples (A); Primitive mantle normalized trace element patterns (B). Studied samples are depleted in the incompatible HFSEs such as $\mathrm{Nb}$ and $\mathrm{Ti}$ which are relative to the primitive mantle. Normalization values after [28] and [29].

\subsection{Petrographical and Geochemical Variations}

Many researchers have shown that mineralogical and geochemical variations in magmatic suites from volcanic arcs can be produced by either magma mixing (e.g. [46] [47]) or assimilation-fractional crystallization processes [48]-[51]. The fractionation process can be further tested by the field, petrographic and geochemical data.

Clinopyroxene is dominant mafic mineral in the quartz monzogabbro, but it is subordinate in the quartz monzodiorite and totally scarce or disappears in the granodiorite and granite. Green hornblende occurs in most samples from the quartz monzodiorite to felsic rocks, sometimes partly replaced by actinolite and biotite. Thus with increasing content of biotite and quartz, clinopyroxene may disappear or give way to hornblende and biotite. Apatite is not ubiquitous but appears as euhedral crystals of variable size; its modal abundance is less than $0.7 \%$ in the quartz monzodiorite and decreases with increasing silica. K-feldspar and quartz occur throughout; they are interstitial in the quartz monzogabbro and their grain-size and abundances increase from these rocks to the granites. Given the wide range of compositions, the lack of disequilibrium minerals, these progressive changes are interpreted as due to crystal fractionation rather than mixing between a mantle-derived basaltic magma and a crustal granitic magma; to this we may add the gradational internal contacts and the lack of mafic enclaves in the more evolved rocks.

Magma mixing and/or mingling and assimilation has frequently been observed in calc alkaline magmatic arc complexes (e.g. [52]) but cannot be invoked to account for the large scale compositional variations seen in the Neshveh intrusion. Although magma mixing can result in linear variations in Harker diagrams, it cannot explain the inflected trends shown by $\mathrm{Na}_{2} \mathrm{O}, \mathrm{Al}_{2} \mathrm{O}_{3}, \mathrm{Sr}$, Eu and $\mathrm{Y}$ (Figure 10 and Figure 11). Finally, minor and trace element abundances plotted in multi-element and rare earth element diagrams (Figure 12) show similar and smoothprogression from one rock type to the next within the intrusion, which is interpreted as resulting from crystal fractionation of the quartz monzogabbros.

A method exhibited by [53] to recognition of the three main processes of partial melting, fractional crystallization and magma mixing in the magmas. In this method, an incompatible element is used against a consistent element. Crystal fractionation is more effective at fractionating compatible elements and discrimination between these two mechanisms may be based on the behavior of trace elements in a logarithmic plot of an incompatible element against a compatible element, where they have very different bulk partition coefficients. In such a diagram, liquids produced by crystal fractionation give a straight line with strong decrease in the compatible element whereas the concentration of the incompatible element $(\mathrm{D}<<1)$ increases slowly; the opposite relationships apply to liquids produced by partial melting [53]. Figure 10 shows that $\mathrm{V}$ content decreases with increasingSiO $\mathrm{S}_{2}$, thus demonstrating its compatible behavior, whereas positive correlations point to the incompatible behavior of $\mathrm{Rb}$ and $\mathrm{Ba}$ (Figure 10). In the log-log plots of $\mathrm{V}$ (compatible element) vs. $\mathrm{Rb}$ and $\mathrm{Ba}$ (incompatible element) (Figure 13), the trends shown are sub-vertical with drastic reduction of the concentration of compatible element (V) throughout the quartz monzogabbro to granodiorite sequence, and the incompatible element contents only increasing rapidly in the granites. This suggests that the main mechanism of differentiation is crystal fractionation.

Although the Neshveh intrusion has various petrographic and mineralogical characteristics, they show similar REE patterns, especially in the HREE (Figure 12). The widely varying concentrations of Nb, Ta and Th (4.3 - 

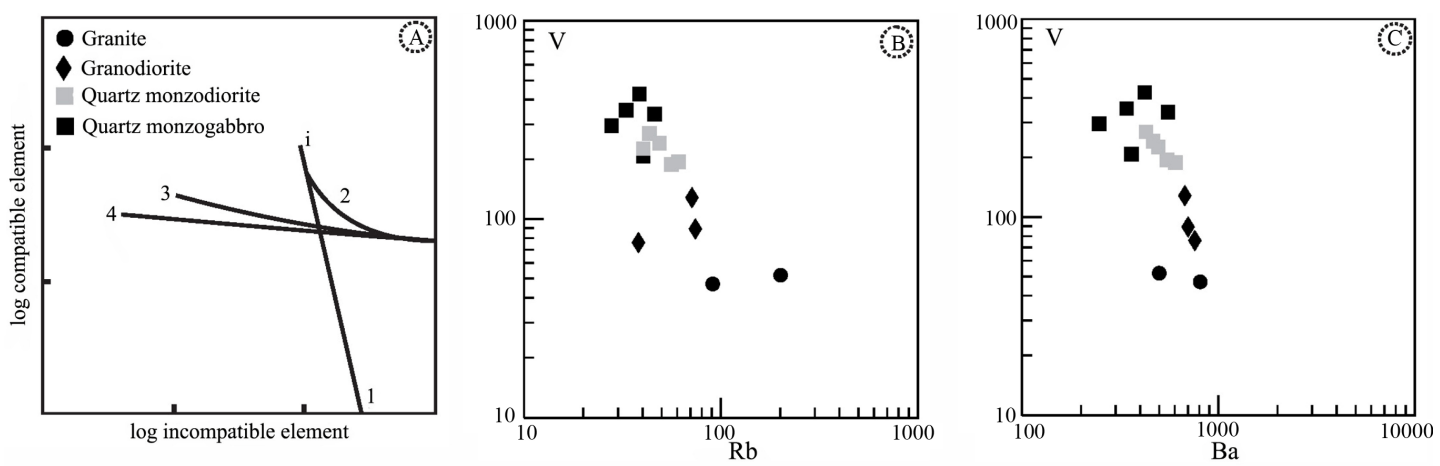

Figure 13. (A) Logarithmic evolution of concentrations for an incompatible element vs. a compatible element during crystal fractionation (1), batch partial melting (2), aggregate melting or fractional fusion with extraction of the mixed melts (3) and fractional fusion with continual removing of the melt formed (4). The initial material is (i) [53]; (B) and (C) Plots for the Neshveh intrusion samples showing near-vertical trends that suggest the main mechanism of differentiation is crystal fractionation.

9.9 ppm, 0.3 - 0.8 ppm, 2.7 - 21.1 ppm, respectively) and gradual changes in Eu-anomaly are also key features. The hypothesis that provides the most satisfactory explanation of thesefeatures is a crystal fractionation model, in which all the rocks were derived from a parental magma via the fractionation. The granitic rocks of the intrusion are the most fractionated rocks, enriched in large-ion lithophile elements ( $\mathrm{Rb}, \mathrm{Tb}, \mathrm{U}$, and $\mathrm{K}$ ) and depleted in $\mathrm{Sr}, \mathrm{P}$ and Ti compared to the others.

\subsection{Mineral Controls}

A general crystal fractionation trend within the representative samples is indicated by decreasing $\mathrm{TiO}_{2}, \mathrm{MgO}_{\text {, }}$ $\mathrm{Fe}_{2} \mathrm{O}_{3}, \mathrm{CaO}$ and $\mathrm{P}_{2} \mathrm{O}_{5}$ concentrations, and increasing $\mathrm{K}_{2} \mathrm{O}$ together with most of the trace elements, e.g., $\mathrm{Ba}, \mathrm{Rb}$, $\mathrm{La}$ and $\mathrm{Ce}$ (Figure 10 and Figure 11). Some elements such as $\mathrm{Na}_{2} \mathrm{O}, \mathrm{Al}_{2} \mathrm{O}_{3}$, $\mathrm{Sr}$ and $\mathrm{Y}$ define broken or curved trends (Figure 10 and Figure 11), a characteristic that allows us to discount their derivation by mixing and/or mingling mechanisms, and instead indicates that they result from crystal fractionation. In order to determine the magmatic evolution of the Neshveh intrusion, the modal mineralogical and chemical compositions are used to model the role of minerals leading to chemical variations in the evolving magma.

The average mode of clinopyroxene is $23 \%$ in the quartz monzogabbro, falling to zero in the granites (Table 1). At the same time, $\mathrm{CaO}$ decreases (Figure 10), suggesting removal of Ca-rich phases. Three Ca bearing minerals in the Neshveh intrusion rocks are plagioclase, clinopyroxene and hornblende. $\mathrm{Sr}$ is a compatible trace element in plagioclase but not in clinopyroxene, so that fractionation of plagioclase causes decreasing $\mathrm{Sr}$ with increasingsilica content [27]. In the Sr vs. MgO plot (Figure 14(A)), Sr increases with increasing MgO up to 3.1 wt\% (corresponding to $55 \mathrm{wt} . \% \mathrm{SiO}_{2}$ ) and then decreases. Since there is no hornblende in the rocks with $\mathrm{Si02}$ less than $55 \mathrm{wt} \%$ (Table 1), it seems thatclinopyroxene had the main role in decreasing concentrations of, e.g., $\mathrm{MgO}, \mathrm{Fe}_{2} \mathrm{O}_{3}, \mathrm{CaO}$ in the rocks with $\mathrm{MgO}>3.1 \mathrm{wt} . \%$. In the rocks with $\mathrm{MgO}<3.1 \mathrm{wt} \%$, magmatic evolution could have been controlled by fractionation of clinopyroxene, plagioclase and hornblende. In the log-log diagram of Rb vs. Sr (Figure 14(B)) [54], it appears that Sr concentration increases from about 350 to $590 \mathrm{ppm}$ as $\mathrm{Rb}$ increases to about $40 \mathrm{ppm}$ in the quartz monzodiorite, and then decreases to $150 \mathrm{ppm}$ in the Rb-rich granite. This can be explained by crystallization of clinopyroxene in the early stages followed by crystallization of the plagioclase, clinopyroxene and hornblende together in the later stage.

Hornblende appears in the Neshveh intrusion phases with $\mathrm{SiO}_{2}>55 \mathrm{wt} \%$ (Table 1). Its mode increases from about $7 \%$ to $16.3 \%$ in the quartz monzodiorite, and then decreases to $2.3 \%$ in the granite. $\mathrm{Y}$ and $\mathrm{Yb}$ are commonly incompatible elements when garnet and hornblende are absent [55] [56]. A significant decrease in Dy/Yb ratio with increasing silica is attributable to removal of hornblende and titanite [57]. In the Neshveh intrusion, Y concentration and $\mathrm{Dy} / \mathrm{Yb}$ ratio remain fairly constant up to $62 \mathrm{wt} \% \mathrm{SiO}_{2}$ and then decrease which is indicating the onset of hornblende and/or titanite fractionation (Figure 11 and Figure 15(A)). The data in Figure 15(B) display a combined vector of hornblende and plagioclase fractionation, suggesting that both played a significant role during magmaticdifferentiation. Considering the modal mineralogical compositions and geochemical re- 

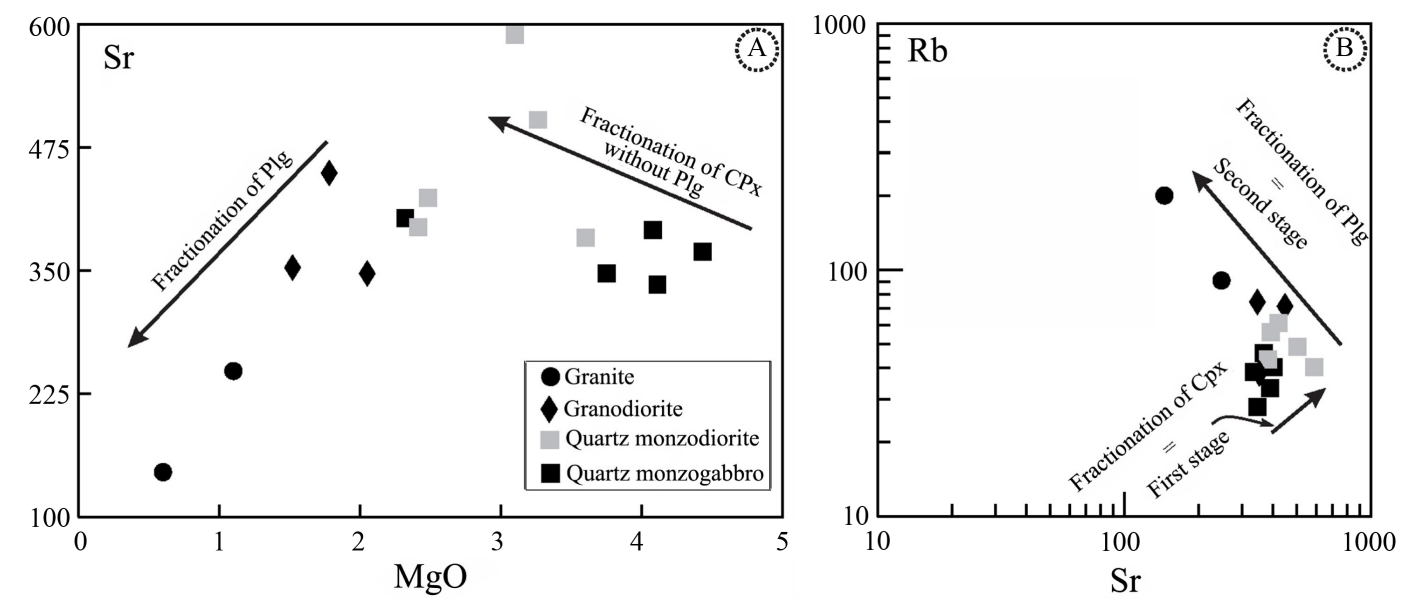

Figure 14. (A) Sr vs. MgO plot showing that as Mg decreases, Sr increases. This relationship suggests plagioclase did not fractionate together with clinopyroxene in the rocks with $\mathrm{MgO}>3.1 \mathrm{wt} \%$; if it had, $\mathrm{Sr}$ as a compatible element in plagioclase, would decrease as MgO decreases [27]; (B) Rb vs. Sr diagram (logarithmic scale) showing the variation of Neshveh intrusion samples (solid vectors are Rayleigh fractionation trends after [54]).
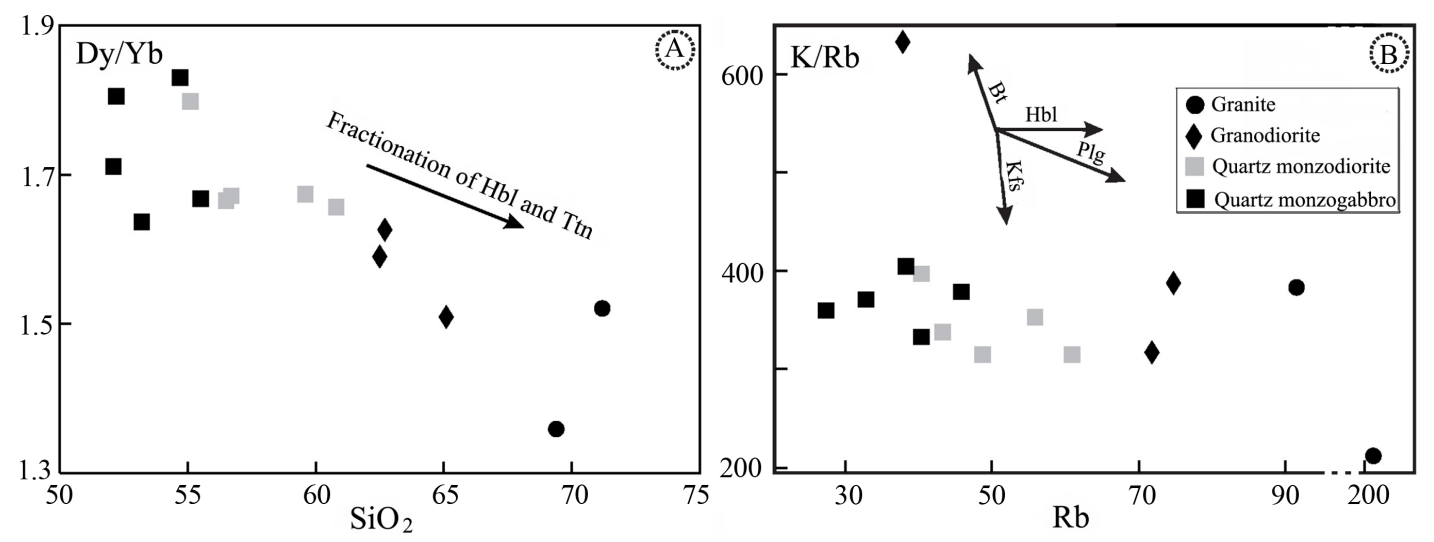

Figure 15. The Neshveh intrusion samples follow the fractionated trends of hornblende on the Dy/Yb vs. $\mathrm{SiO}_{2}$ (A) and Kf/Rb vs. Rb (B) diagrams.

sults, hornblende fractionation only played a role in the formation of rocks with more than $62 \mathrm{wt} \% \mathrm{SiO}_{2}$ and consequently caused decrease of $\mathrm{CaO}, \mathrm{MgO}$ and $\mathrm{FeO}$ in the magma.

The role of plagioclase is best examined through $\mathrm{Na}_{2} \mathrm{O}$, $\mathrm{Sr}$ and Eu trends in the representative samples. $\mathrm{Na}_{2} \mathrm{O}$ shows an inflected trend with increasing silica content, increasing up to $55 \mathrm{wt} \% \mathrm{SiO}_{2}$ and then decreasing (Figure 10). $\mathrm{Sr}$ and Eu substitute for $\mathrm{Ca}$ and $\mathrm{Na}$ in plagioclase (but not in clinopyroxene); they have inflected trends in these samples that mimic that of $\mathrm{Na}_{2} \mathrm{O}$. These trends can be interpreted as indicating that plagioclase fractionation was more important in the formation of rocks with $\mathrm{SiO}_{2}>55 \mathrm{wt} \%$ compared to the rocks with $\mathrm{SiO}_{2}<55$ wt\% (petrographic observations show that there is no significant change in the proportion of plagioclase up to this point, Table 1). This is also the only reasonable explanation for the development of negative Eu anomalies in the granites. Thus, plagioclase would have had no effect on $\mathrm{CaO}, \mathrm{Na}_{2} \mathrm{O}$ and $\mathrm{Al}_{2} \mathrm{O}_{3}$ in the less siliceous rocks.

$\mathrm{Ba} / \mathrm{Sr}$ ratio will help to identify the relative roles of K-feldspar and plagioclase, since it increases with precipitation of plagioclase from the magma, but decreases when K-feldspar and biotite start to precipitate [58]. In the Neshveh intrusion rocks, $\mathrm{Ba} / \mathrm{Sr}$ is constant up to $55 \mathrm{wt} \% \mathrm{SiO}_{2}$ and then increases, showing the effect of plagioclase precipitation (Figure 16). Ba concentration increases with increasing $\mathrm{SiO}_{2}$ without any inflection (Figure 11), suggesting that K-feldspar and biotite are late-crystallized minerals and/or sank very slowly in the co-existing melt during magmatic evolution [59]. Essentially, constant $\mathrm{K} / \mathrm{Rb}$ ratios (Figure 14(B)) and the positive correlation between $\mathrm{K}_{2} \mathrm{O}$ and $\mathrm{SiO}_{2}$ (Figure 11) are also consistent with no K-feldspar removal. In addition, 


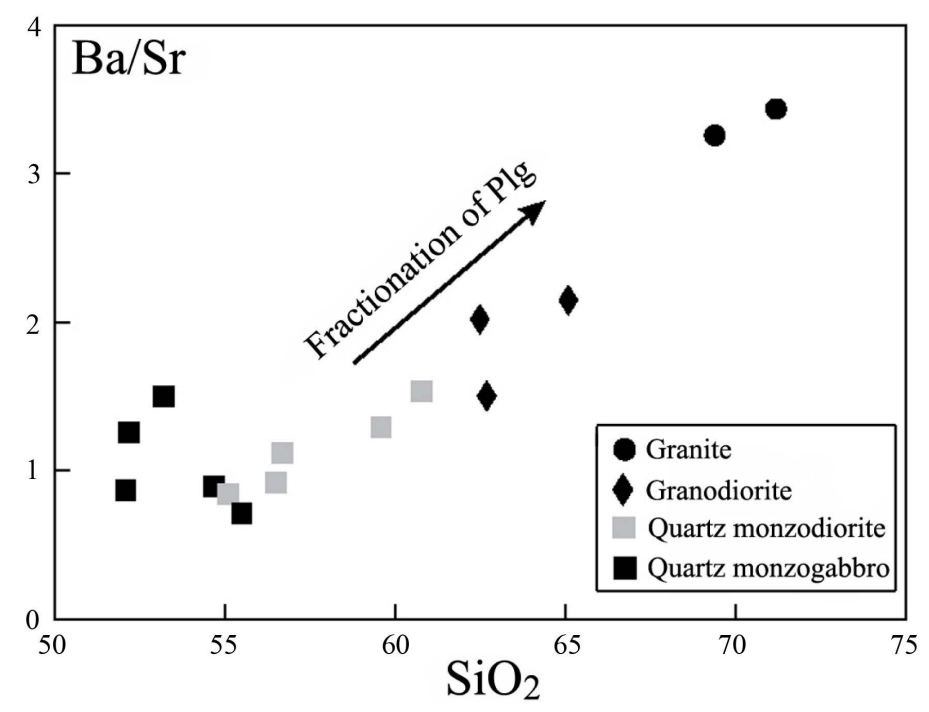

Figure 16. Increasing $\mathrm{Ba} / \mathrm{Sr}$ vs. $\mathrm{SiO}_{2}$ indicates fractionation of plagioc1ase rather than K-feldspar in the Neshveh intrusion.

the lack of K-feldspar and biotite fractionations are also confirmed by the absence of a negative Ba anomaly in the primitive mantlenormalized rare earth element patterns (Figure 12(B)).

Fractionation of biotite and K-feldspar should buffer or reduce $\mathrm{Ba}$ in the residual liquid [60]. $\mathrm{K}_{2} \mathrm{O}$ and $\mathrm{Ba}$ contents increase from $1.21 \mathrm{wt} \%$ and $247 \mathrm{ppm}$, respectively, in quartz monzogabbro to $5.14 \mathrm{wt} \%$ and $808 \mathrm{ppm}$ in granite (Figure 10 and Figure 11; Table 2), indicating that biotite and K-feldspar crystallized late or sank very slowly in co-existing melt during magmatic differentiation. As presented in Table 1, biotite content decreases in the granitic samples, thus increasing of $\mathrm{Ba}$ in these samples is mostly related to late-crystallize $\mathrm{K}$ feldspar in the magma.

The regular decrease in $\mathrm{P}_{2} \mathrm{O}_{5}$ content from the quartz monzogabbroic to granitic rocks (Figure 10) is attributed to fractionation of apatite [9].

Ti-bearing minerals such as ilmenite and titanite might be other fractionated phases in the Neshveh intrusion, as indicated by the decrease of $\mathrm{TiO}_{2}$ with increasing $\mathrm{SiO}_{2}$ (Figure 10). Moreover, as hornblende and biotite fractionated from the magma forming rocks with $\mathrm{SiO}_{2}>\sim 62 \mathrm{wt} \%$, a combination of ilmenite, titanite, hornblende and biotite fractionations can be considered responsible for the decreasing $\mathrm{TiO}_{2}$ in granodioritic and granitic melts. There is a larger negative Ti anomaly in the granitic rocks and decreasing $\mathrm{Dy} / \mathrm{Yb}$ ratio vs. $\mathrm{SiO}_{2}$ in the representative samples (Figure 12(B) and Figure 15(A)).

\section{Conclusion}

Field studies along with petrographic and geochemical investigations indicate that the Neshveh intrusion composed from the quartz monzogabbro, quartz monzodiorite, granodiorite and granite. The wide compositional and mineralogical range of the Neshveh intrusion, from quartz monzogabbro through to granite, is typical of a calc alkaline arc intrusion. This intrusion has high-K calc-alkaline nature classified as I-type granitoids. All phases of the Neshveh granitoid are characterized by LREE-rich patterns with high LREE/HREE ratio and negative Eu anomalies. Similarity of patterns suggests a comagmatic source for these rocks and demonstrates the role of magmatic differentiation in their evolution. Field relations, together with mineral composition and geochemical studies demonstrate that all rock types are co-magmatic and related to a single parent magma which is underwent fractional crystallization. This process commonly present as mineral assemblage at different stages, clinopyroxene in the quartz monzogabbro, clinopyroxene, hornblende and plagioclase in the quartz monzodiorite, and hornblende, plagioclase \pm biotite thereafter. K-Feldspar, biotite and quartz are progressively concentrated in the granodiorite and granite, but did not separate from them in large amounts until the final stage of $\mathrm{SiO}_{2}$ enrichment. The initial magma for the Neshveh intrusion was probably similar to the most mafic rock type exposed, the quartz monzogabbro, with about $52 \mathrm{wt} \% \mathrm{SiO}_{2}$. 


\section{Acknowledgements}

This study was financially support by Research Deputy of Tehran University for the proposal entitled Petrography and Petrology of Neshveh intrusion (northwest Saveh). Our gratitude is expressed to their helps.

\section{References}

[1] Dercourt, J., Zonenshain, L.P., Ricou, L.E., Kazmin, V.G., Pichon, X.L., Knipper, A.L., Grandjacquet, C., Sbortshikov, I.M., Geyssant, J., Lepvrier, C., Pechersky, D.H., Boulin, J., Sibuet, J.C., Savostin, L.A., Sorokhtin, O., Westphal, M., Bazhenov, M.L., Lauer, J.P. and Biju-Duval, B. (1986) Geological Evolution of the Tethys Belt from the Atlantic to the Pamirs Since the LIAS. Tectonophysics, 123, 241-315. http://dx.doi.org/10.1016/0040-1951(86)90199-X

[2] Ricou, L.E., Braud, J. and Brunn, J.H. (1977) Le Zagros. Socie’te Geologique de France. Me'moires, 8, 33-52.

[3] Agard, P., Jolivet, L., Vrielynck, B., Burov, E. and Monié, P. (2007) Plate Acceleration: The Obduction Trigger? Earth and Planetary Science Letters, 258, 428-441. http://dx.doi.org/10.1016/j.epsl.2007.04.002

[4] McQuarrie, N., Stock, J.M., Verdel, C. and Wernicke, B.P. (2003) Cenozoic Evolution of Neotethys and Implications for the Causes of Plate Motions. Geophysical Research Letters, 30, 20-36. http://dx.doi.org/10.1029/2003GL017992

[5] Talebian, M. and Jackson, J. (2004) A Reappraisal of Earthquake Local Mechanisms and Active Shortening in the Zagros Mountain of Iran. Geophysical Journal International, 156, 506-526. http://dx.doi.org/10.1111/j.1365-246X.2004.02092.x

[6] Vernant, P., Nilforoushan, F., Hatzfeld, D., Abbassi, M.R, Vigny, C., Masson, F., Nankali, H., Martinod, J., Ashtiani, A., Bayer, R., Tavakoli, F. and Chery, J. (2004) Present-Day Crustal Deformation and Plate Kinematics in the Middle East Constrained by GPS Measurements in Iran and Northern Oman. Geophysical Journal International, 157, 381-398. http://dx.doi.org/10.1111/j.1365-246X.2004.02222.x

[7] Molinaro, M., Guezou, J.C., Leturmy, P., Eshraghi, S.A. and de Lamotte, D.F. (2004) The Origin of Changes in Structural Style across the Bandar Abbas Syntaxis, SE Zagros (Iran). Marine and Petroleum Geology, 21, 735-752. http://dx.doi.org/10.1016/j.marpetgeo.2004.04.001

[8] Molinaro, M., Zeyen, H. and Laurencin, X. (2005) Lithospheric Structure beneath the South-Eastern Zagros Mountains, Iran: Recent Slab Break-Off? Terra Nova, 17, 1-6. http://dx.doi.org/10.1111/j.1365-3121.2004.00575.x

[9] Meyer, B., Mouthereau, F., Lacombe, O. and Agard, P. (2005) Evidence for Quaternary Activity along the Dehshir Fault: Implication. Geophysical Journal International, 163, 1-10.

[10] Hassanzadeh, J. (1993) Metallogenic and Tectonomagmatic Events in the SE Sector of the Cenozoic Active Continental Margin of Central Iran. Unpublished Ph.D. Thesis, University of California, Los Angeles, 204 p.

[11] Torabi, G. (2009) Subduction-Related Eocene Shoshonites from the Cenozoic Urumieh-Dokhtar Magmatic Arc (Qaleh-Khargooshi Area, Western Yazd Province, Iran). Turkish Journal of Earth Sciences, 18, 1-34.

[12] Caillat, C., Dehlavi, P. and Martel, J.B. (1978) Geologie de la region de Saveh (Iran). Contribution a l'etude du volcanism et du plutonism tertiaresde la zone de I Irancentral. Ph.D. Thesis, Grenoble, 325 p.

[13] Helmi, F. (1991) Petrology and Geochemistry of Igneous Rocks in Niousht Area (NE Saveh). M.Sc. Thesis, University of Tehran, Tehran. (in Persian)

[14] Panahi, A., Keshavarzi, R., Kiani, M., Taheri, M. and Javadian, B. (2013) Petrography and Petrogenesis of the Neshveh Intrusive Rocks, Northeast Saveh, Central Iran. Journal of Academic and Applied Studies (Special Issue on Applied Sciences), 3, 22-37.

[15] Keshavarzi, R., Esmaili, D., Kahkhaie, M.R., Jabari, R. and Mokhtari, M.A.A. (2014) Petrology, Geochemistry and Tectonomagmatic Setting of Neshveh Intrusion (NW Saveh). Open Journal of Geology, 4, 177-189. http://dx.doi.org/10.4236/ojg.2014.45013

[16] Aghanabati, S.A. (2004) Geology of Iran. Geological Survey of Iran. 606 p. (in Persian)

[17] Galamgash, J. and Fonudi, M. (1998) Explanatory Text of Saveh. Geological Map 1:100000, Geological Survey of Iran, Tehran.

[18] Davarpanah, A. (2009) Magmatic Evolution of Eocene Volcanic Rocks of the Bijgerd-Kuh-e-Kharchin Area, OrumiehDokhtar Zone, Iran. M.Sc. Thesis, Georgia State University, Atlanta.

[19] Middlemost, E.A.K. (1985) Magmas and Magmatic Rocks. An Introduction to Igneous Petrology. Longman Group Ltd., London, New York, 266 p.

[20] Kretz, R. (1983) Symbols for Rock-Forming Minerals. American Mineralogist, 68, 277-279.

[21] Morimoto, N. (1988) Nomenclature of Pyroxenes: Subcommittee on Pyroxenes, Commission on New Minerals and Mineral Names, International Mineralogical Association. American Mineralogist, 73, 1123-1133. 
[22] Leake, B.E. (1997) Nomenclature of Amphiboles: Report of the Subcommittee on Amphiboles of the International Mineralogical Association, Commission on New Minerals and Mineral Names. Mineralogical Magazine, 61, 295-321.

[23] Rickwood, P.C. (1989) Boundary Lines within Petrologic Diagrams Which Use Oxides of Major and Minor Elements. Lithos, 22, 247-263. http://dx.doi.org/10.1016/0024-4937(89)90028-5

[24] Chappell, B.W. and White, A.J.R. (1992) I- and S-Type Granites in the Lachlan Fold Belt. Transactions of the Royal Society of Edinburgh, Earth Sciences, 83, 1-26.

[25] Chappell, B.W. and White, A.J.R. (2001) Two Contrasting Granite Types. 25 Years Later. Australian Journal of Earth Sciences, 48, 489-499. http://dx.doi.org/10.1046/j.1440-0952.2001.00882.x

[26] Chappell, B.W. and White, A.J.R. (1983) Granitoid Types and Their Distribution in the Lachlan Fold Belt, Southeastern Australia. Geological Society of America Memoirs, 159, 21-37. http://dx.doi.org/10.1130/MEM159-p21

[27] Wilson, M. (2007) Igneous Petrogenesis. Chapman and Hall, London, 411 p.

[28] Boynton, W.V. (1984) Cosmochemistry of the Rare Earth Elements: Meteorite Studies. In: Henderson, P., Eds., Rare Earth Element Geochemistry, Elsevier, Amsterdam, 63-114.

[29] Sun, S.S. and McDonough, W.F. (1989) Chemical and Isotopic Systematics of Oceanic Basalts: Implications for Mantle Composition and Processes. In: Saunders, A.D. and Norry, M.J., Eds., Magmatism in the Ocean Basins, in: Geological Society Special Publication, Vol. 42, 313-345.

[30] Schmidt, A., Weyer, S. and Brey, G.P. (2006) BSE Reservoirs: Insights from Nb/Ta of Rutile-Bearing Eclogites. Geochimica et Cosmochimica Acta, 70, A562

[31] Glenn, A.G. (2004) The Influence of Melt Structure on Trace Element Partitioning Near the Peridotite Solidus. Contribution to Mineralogy and Petrology, 147, 511-527. http://dx.doi.org/10.1007/s00410-004-0575-1

[32] Sajona, F.G., Maury, R.C., Bellon, H., Cotton, J. and Defant, M. (1996) High Field Strength Elements of PliocenePleistocene Island Arc Basalts Zamboanga Peninsula, Western Mindanao (Philippines). Journal of Petrology, 37, 693726. http://dx.doi.org/10.1093/petrology/37.3.693

[33] Chappell, B.W. (1999) Aluminium Saturation in I and S-Type Granites and the Characterization of Fractionated Haplogranites. Lithos, 46, 535-551. http://dx.doi.org/10.1016/S0024-4937(98)00086-3

[34] Kamber, B.S., Ewart, A., Collerson, K.D., Bruce, M.C. and McDonald, G.D. (2002) Fluid-Mobile Trace Element Constraints on the Role of Slab Melting and Implications for Archean Crustal Growth Models. Contribution to Mineralogy and Petrology, 144, 38-56. http://dx.doi.org/10.1007/s00410-002-0374-5

[35] Atherton, M.P. and Ghani, A.A. (2002) Slab Breakoff: A Model for Caledonian, Late Granite Syn-Collisional Magmatism in the Orthotectonic (Metamorphic) Zone of Scotland and Donegal, Ireland. Lithos, 62, 65-85. http://dx.doi.org/10.1016/S0024-4937(02)00111-1

[36] Keshavarzi, R. (2009) Petrography and Petrology of Neshveh Granitoid (Northwest Saveh). Unpublished M.Sc. Thesis in Petrology, University of Tehran, Tehran. (in Persian)

[37] Eichelberger, J.C. (1980) Vesiculation of Mafic Magma during Replenishment of Silicic Magma Reservoirs. Nature, 288, 446-450. http://dx.doi.org/10.1038/288446a0

[38] Hildreth, W. (1981) Gradients in Silicic Magma Chambers: Implications for Lithospheric Magmatism. Journal of Geophysical Research: Solid Earth, 86, 10153-10192. http://dx.doi.org/10.1029/JB086iB11p10153

[39] Furlong, K.P. and Fountain, D.M. (1986) Continental Crustal Underplating: Thermal Considerations and Seismic-Petrologic Consequences. Journal of Geophysical Research: Solid Earth, 91, 8285-8294. http://dx.doi.org/10.1029/JB091iB08p08285

[40] Arndt, N.T. and Goldstein, S.L. (1989) An Open Boundary between Lower Continental Crust and Mantle: Its Role in Crust Formation and Crustal Recycling. Tectonophysics, 161, 201-212. http://dx.doi.org/10.1016/0040-1951(89)90154-6

[41] Bergantz, G.W. (1989) Underplating and Partial Melting: Implications for Melt Generation and Extraction. Science, 245, 1093-1095. http://dx.doi.org/10.1126/science.245.4922.1093

[42] Chappell, B.W., White, A.J.R. and Wyborn, D. (1987) The Importance of Residual Source Material (Restite) in Granite Petrogenesis. Journal of Petrology, 28, 1111-1138. http://dx.doi.org/10.1093/petrology/28.6.1111

[43] Roberts, M.P. and Clemens, J.D. (1995) Feasibility of AFC Models for the Petrogenesis of Calc-Alkaline Magma Series. Contributions to Mineralogy and Petrology, 121, 139-147. http://dx.doi.org/10.1007/s004100050095

[44] Sha, L.K. and Chappell, B.W. (1999) Apatite Chemical Composition, Determined by Electron Microprobe and LaserAblation Inductively Coupled Plasma Mass Spectrometry, as a Probe into Granite Petrogenesis. Geochimica et Cosmochimica Acta, 63, 3861-3881. http://dx.doi.org/10.1016/S0016-7037(99)00210-0

[45] Broska, I., Williams, C.T., Uher, P., Konečný, P. and Leichmann, J. (2004) The Geochemistry of Phosphorus in Dif- 
ferent Granite Suites of the Western Carpathians, Slovakia: The Role of Apatite and P-Bearing Feldspar. Chemical Geology, 205, 1-15. http://dx.doi.org/10.1016/j.chemgeo.2003.09.004

[46] Popov, V.S., Tevelev, A.A. and Bogatov, V.I. (1999) The Stepninsk Pluton on the South Urals: Relationships of Plutonic Rocks Coming from Mantle and Crustal Sources. Izvestiya Vuzov Geologiya i Razvedka Jurnal, 5, 52-68.

[47] Bea, F., Fershtater, G.B., Montero, P., Smirnov, V.N. and Molina, J.F. (2005) Deformation-Driven Differentiation of Granitic Magma: The Stepninsk Pluton of the Uralides, Russia. Lithos, 81, 209-233. http://dx.doi.org/10.1016/j.lithos.2004.10.004

[48] DePaolo, D.J. (1981) Trace Element and Isotopic Effects of Combined Wall Rock Assimilation and Fractional Crystallization. Earth and Planetary Science Letters, 53, 189-202. http://dx.doi.org/10.1016/0012-821X(81)90153-9

[49] Spera, F.J. and Bohrson, W.A. (2001) Energy-Constrained Open System Magmatic Processes I: General Model and Energy-Constrained Assimilation and Fractional Crystallization (EC-AFC) Formulation. Journal of Petrology, 42, 9991018. http://dx.doi.org/10.1093/petrology/42.5.999

[50] Thompson, A.B., Matile, L. and Ulmer, P. (2002) Some Thermal Constraints on Crustal Assimilation during Fractionation of Hydrous, Mantle-Derived Magmas with Examples from Central Alpine Batholiths. Journal of Petrology, 43, 403-422. http://dx.doi.org/10.1093/petrology/43.3.403

[51] Kuritani, T., Kitagawa, H. and Nakamura, E. (2005) Assimilation and Fractional Crystallization Controlled by Transport Process of Crustal Melt: Implications from an Alkali Basalt-Dacite Suite from Rishiri Volcano, Japan. Journal of Petrology, 46, 1421-1442. http://dx.doi.org/10.1093/petrology/egi021

[52] Chappell, B.W. (1996) Magma Mixing and the Production of Compositional Variation within Granite Suites: Evidence from the Granites of Southeastern Australia. Journal of Petrology, 37, 449-470. http://dx.doi.org/10.1093/petrology/37.3.449

[53] Cocherie, A. (1986) Systematic Use of Trace Element Distribution on Patterns in Log-Log Diagrams. Geochimica et Cosmoshimica Acta, 50, 2517-2522. http://dx.doi.org/10.1016/0016-7037(86)90034-7

[54] Klimm, K., Holtz, F. and King, P.L. (2008) Fractionation vs. Magma Mixing in the Wangrah Suite A-Type Granites. Lachlan Fold Belt. Australia: Experimental Constraints. Lithos, 102, 415-434.

[55] Green, D.H. (1980) Island Arc and Continent-Building Magmatism—A Review of Petrogenic Models Based on Experimental Petrology and Geochemistry. Tectonophysics, 63, 367-385. http://dx.doi.org/10.1016/0040-1951(80)90121-3

[56] Winter, J.D. (2001) An Introduction to Igneous and Metamorphic Petrology. Prentice Hall, Upper Saddle River.

[57] Davidson. J., Turner, S., Handley, H., Macpherson, C. and Dosseto, A. (2007) Amphibole “Sponge” in Arc Crust? Geology, 35, 787-790. http://dx.doi.org/10.1130/G23637A.1

[58] Rollinson, H.R. (1993) Using Geochemical Data: Evaluation, Presentation and Interpretation. Longman, Harlow, 352 p.

[59] Wyborn, D., Chappell, B.W. and James, M. (2001) Examples of Convective Fractionation in High Temperature Granites from the Lachlan Fold Belt. Australian Journal of Earth Sciences, 48, 531-541. http://dx.doi.org/10.1046/j.1440-0952.2001.00877.x

[60] Blundy, J. and Wood, B. (2003) Partitioning of Trace Elements between Crystals and Melts. Earth and Planetary Science Letters, 210, 383-397. http://dx.doi.org/10.1016/S0012-821X(03)00129-8 